\title{
Beyond the electric-dipole approximation in simulations of x-ray absorption spectroscopy: Lessons from relativistic theory
}

Cite as: J. Chem. Phys. 152, 184110 (2020); https://doi.org/10.1063/5.0003103

Submitted: 30 January 2020 . Accepted: 09 April 2020 . Published Online: 13 May 2020

Nanna Holmgaard List (D), Timothé Romain Léo Melin (D), Martin van Horn (D), and Trond Saue (D)

\section{ARTICLES YOU MAY BE INTERESTED IN}

ReSpect: Relativistic spectroscopy DFT program package

The Journal of Chemical Physics 152, 184101 (2020); https://doi.org/10.1063/5.0005094

Essentials of relativistic quantum chemistry

The Journal of Chemical Physics 152, 180901 (2020); https://doi.org/10.1063/5.0008432

PSI4 1.4: Open-source software for high-throughput quantum chemistry

The Journal of Chemical Physics 152, 184108 (2020); https://doi.org/10.1063/5.0006002

\section{Lock-in Amplifiers up to $600 \mathrm{MHz}$}
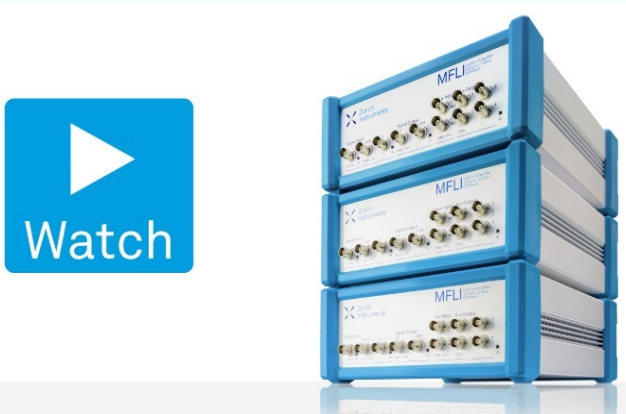


\title{
Beyond the electric-dipole approximation in simulations of $\mathrm{x}$-ray absorption spectroscopy: Lessons from relativistic theory
}

\author{
Cite as: J. Chem. Phys. 152, 184110 (2020); doi: 10.1063/5.0003103
}

Submitted: 30 January 2020 - Accepted: 9 April 2020 •

Published Online: 13 May 2020

\author{
Nanna Holmgaard List, ${ }^{1,2, a)}$ (D) Timothé Romain Léo Melin, ${ }^{3, b)}$ (D) Martin van Horn, ${ }^{3}$ (D) and Trond Saue ${ }^{3, c)}$ (D) \\ AFFILIATIONS \\ ${ }^{1}$ Department of Chemistry and the PULSE Institute, Stanford University, Stanford, California 94305, USA \\ ${ }^{2}$ SLAC National Accelerator Laboratory, Menlo Park, California 94025, USA \\ ${ }^{3}$ Laboratoire de Chimie et Physique Quantique, UMR 5626 CNRS_Université Toulouse III-Paul Sabatier, 118 Route de Narbonne, \\ F-31062 Toulouse, France \\ a) Electronic mail: nhlist@stanford.edu \\ b) Present address: Department of Chemistry, Michigan State University, East Lansing, Michigan 48824, USA. \\ ${ }^{c}$ Author to whom correspondence should be address: trond.saue@irsamc.ups-tlse.fr. URL: http://dirac.ups-tlse.fr/saue
}

\begin{abstract}
We present three schemes to go beyond the electric-dipole approximation in x-ray absorption spectroscopy calculations within a fourcomponent relativistic framework. The first is based on the full semi-classical light-matter interaction operator and the two others on a truncated interaction within the Coulomb gauge (velocity representation) and multipolar gauge (length representation). We generalize the derivation of the multipolar gauge to an arbitrary expansion point and show that the potentials corresponding to different expansion points are related by a gauge transformation, provided that the expansion is not truncated. This suggests that the observed gauge-origin dependence in the multipolar gauge is more than just a finite-basis set effect. The simplicity of the relativistic formalism enables arbitrary-order implementations of the truncated interactions, with and without rotational averaging, allowing us to test their convergence behavior numerically by comparison to the full formulation. We confirm the observation that the oscillator strength of the electric-dipole allowed ligand $K$-edge transition of $\mathrm{TiCl}_{4}$, when calculated to the second order in the wave vector, becomes negative but also show that inclusion of higher-order contributions allows convergence to the result obtained using the full light-matter interaction. However, at higher energies, the slow convergence of such expansions becomes dramatic and renders such approaches at best impractical. When going beyond the electric-dipole approximation, we therefore recommend the use of the full light-matter interaction.
\end{abstract}

Published under license by AIP Publishing. https://doi.org/10.1063/5.0003103

\section{INTRODUCTION}

The importance of relativistic effects in chemistry is illustrated by the fact that without relativity, gold would have the same color as silver, ${ }^{1-3}$ mercury would not be liquid at room temperature, ${ }^{4,5}$ and your car, if using a lead battery, would not start. ${ }^{6}$ The present work highlights another aspect of relativity, namely, its essential role in light-matter interactions.

A semi-classical treatment invoking the electric-dipole (ED) approximation is a common starting point for a theoretical description of light-matter interactions. The latter approximation assumes that the spatial extent of the molecular system is small compared to the wavelength of the electromagnetic field such that the molecule effectively sees a uniform electric field, while the magnetic field component is neglected. Formally, it corresponds to retaining only the zeroth-order term of an expansion of the interaction operator in orders of the length of the wave vector. While this is often welljustifiable for the most commonly used optical laser sources and intensities, the availability of (i) high-energy x-ray photons, with wavelengths comparable to the molecular target, ${ }^{7-9}$ and (ii) intense laser sources, creating high-energy electrons strongly influenced by the magnetic component of the Lorentz force, ${ }^{10-12}$ motivates investigations into the effects of going beyond this simplification. Clearly, in either limit, relativistic effects become increasingly important, as 
the velocity of the electron being probed or driven by the laser field reaches a substantial fraction of the speed of light.

In this work, we focus on going beyond the electric-dipole (BED) approximation in relativistic simulations of near-edge $\mathrm{x}$-ray absorption spectroscopy. While non-dipolar corrections to the total cross sections first enter at the second order and are generally quite small (5\%-10\% for dipole-allowed $K$-edge transitions in the soft $\mathrm{x}$-ray region, reaching up $\sim 20 \%$ in the hard $x$-ray region ${ }^{8}$ ), the important $K$ pre-edge features may, as is often the case in transition metal complexes, be (near) electric-dipole-forbidden. ${ }^{13-15}$ In general, methods for going beyond the ED approximation have been based on multipole expansions of the minimal coupling light-matter interaction operator, which, in truncated form, may introduce unphysical gauge-origin dependence into the molecular properties. ${ }^{16}$ This is particularly problematic for molecular systems where no natural choice of gauge origin exists. In a seminal paper, Bernadotte et al. presented an approach for the calculation of origin-independent intensities within the non-relativistic framework, beyond the ED approximation, by truncating the oscillator strength, rather than the interaction operator, in orders of the wave vector. ${ }^{17}$ In the velocity representation, they could demonstrate origin independence of oscillator strengths to arbitrary orders and could confirm this by calculation to the second order. Bernadotte et al., furthermore, transformed the interaction operator truncated to the second order in the wave vector from its velocity representation to a multipolar form (for earlier demonstrations of this transformation, see Refs. 18 and 19). This would imply origin independence of oscillator strengths to arbitrary orders also in the length representation, but this was not observed in calculations to the second order and attributed to the finite basis approximation. Further complications were reported by Lestrange et al..$^{20}$ who found that including the second-order oscillator strength of the ED allowed ligand $K$-edge transition of $\mathrm{TiCl}_{4}$ made the total oscillator strength negative. Negative oscillator strengths to the second order were also reported by Sørensen et al. ${ }^{21}$ in metal $K$-edge transitions of $\left[\mathrm{FeCl}_{4}\right]^{-}$, but only for certain basis sets, which led them to conclude that they were due to incomplete basis sets rather than missing higher-order contributions to the oscillator strength. In a second paper, ${ }^{22}$ where $\left[\mathrm{FeCl}_{4}\right]^{-}$is revisited, Sørensen et al. speculated that the fourth-order electric-octupoleelectric-octupole contribution may reverse the sign "provided that no other higher terms also grow disproportionately large."

To avoid the above issues, we recently proposed using the full semi-classical light-matter interaction operator in the context of linear absorption spectroscopy in the non-relativistic regime. ${ }^{8}$ In a Gaussian basis, the necessary integrals over the light-matter interaction operator can be identified as Fourier transforms of overlap distributions, as shown by Lehtola et al. for dynamic structure factors, and can be easily evaluated within standard integral schemes, such as McMurchie-Davidson ${ }^{8}$ or Gauss-Hermite quadrature. ${ }^{24}$ In a second paper, ${ }^{25}$ we presented a mixed analytical-numerical approach to isotropically average oscillator strengths computed with the full light-matter interaction operator. ${ }^{25}$ This novel approach has been followed up by Sørensen et al. ${ }^{24,26}$ Some other works using full lightmatter interaction may be mentioned: Markin and Kaplan calculated the photoionization cross section of the $\mathrm{H}_{2}$ molecule in both a nonrelativistic ${ }^{27}$ and relativistic setting. ${ }^{28}$ More recent works along these lines include Refs. 9, 29, and 30. A recent review has been given by Wang et al. ${ }^{3}$
In the following, we present three schemes for computing linear absorption cross sections beyond the ED approximation within a four-component relativistic framework: (i) the full semi-classical light-matter interaction as well as two approaches based on truncated interaction either using a (ii) multipolar gauge (mg) (length representation) or a (iii) Coulomb gauge (velocity representation). The latter may be viewed as an extension of the work by Bernadotte et $a l .{ }^{17}$ to the relativistic domain. For all three schemes, we present methods for rotational averaging; for the full interaction, we use the mixed analytical-numerical approach already reported for nonrelativistic calculations, ${ }^{25}$ whereas for truncated interaction, we have developed a fully analytical approach. As will become clear below, in addition to providing a more general framework, the relativistic formalism is simpler than the non-relativistic counterpart and facilitates general, easily programmable expressions. In fact, we have in the DIRAC package ${ }^{32}$ implemented the two schemes for truncated interaction to arbitrary orders, with and without rotational averaging. This allows us to test numerically the convergence behavior of these schemes and compare to the formulation based on the full semi-classical light-matter interaction.

The paper is organized as follows: In Sec. II A, we briefly review the description of semi-classical light-matter interactions in both relativistic and non-relativistic frameworks. Section II B presents the working expressions for oscillator strengths for the full lightmatter interaction operator, followed by a derivation of the two different truncated light-matter interaction formulations in Sec. II C. In Sec. II D, we describe schemes for obtaining isotropically averaged oscillator strengths in each of the three cases. In Sec. IV, we investigate the performance of the three different schemes for going beyond the electric-dipole approximation before concluding in Sec. V.

We also provide four appendixes: In Appendix A, we explain how electronic spectra are simulated in the DIRAC package in the framework of time-dependent response theory. In Appendix B, we discuss the multipolar gauge and, contrary to previous works, discuss the gauge transformation between different expansion points. In Appendix $\mathrm{C}$, we present the trivariate beta function, which plays a key role in the fully analytic approach to rotational averaging. Finally, in Appendix D, we give technical details about integrals over full and truncated light-matter interaction in Gaussian- and Slater-type orbitals.

\section{THEORY}

We start by reviewing the theory of interactions of molecules with electromagnetic radiation within a relativistic but semi-classical description before deriving three different schemes for computing oscillator strengths beyond the ED approximation. Finally, we present expressions for isotropically averaged oscillator strengths for each case. The resulting expressions have been implemented in a development version of the DIRAC program.

\section{A. Coupling particles and fields}

External fields are introduced into the Hamiltonian $\hat{H}$ through the substitutions,

$$
\hat{\mathbf{p}} \rightarrow \hat{\boldsymbol{\pi}}=\hat{\mathbf{p}}-q \mathbf{A}, \quad \hat{H} \rightarrow \hat{H}+q \phi,
$$

where $q$ is the particle charge, $\phi$ is the scalar potential, $\mathbf{A}$ is the vector potential, $\hat{\mathbf{p}}$ is the linear momentum, and $\hat{\boldsymbol{\pi}}$ is the mechanical 
momentum. The expectation value of the resulting interaction Hamiltonian may then be expressed as

$$
\left\langle\hat{H}_{\text {int }}\right\rangle=\int \rho(\mathbf{r}, t) \phi(\mathbf{r}, t) d^{3} \mathbf{r}-\int \mathbf{A}(\boldsymbol{r}, t) \cdot \mathbf{j}(\mathbf{r}, t) d^{3} \mathbf{r},
$$

where the scalar potential is seen to couple to the charge density $\rho$ and the vector potential to the current density $\boldsymbol{j}$. The substitutions in Eq. (1) have been termed the principle of minimal electromagnetic coupling ${ }^{33}$ since it only refers to a single property of the particles, namely, charge. Interestingly, it arises from the interaction Lagrangian proposed by Schwarzschild ${ }^{34}$ in 1903, two years before the annus mirabilis of Einstein. The expectation value of the interaction Hamiltonian, Eq. (2), can be expressed compactly in terms of 4-current $j_{\mu}$ and 4-potential $A_{\mu}$,

$$
\left\langle\hat{H}_{\text {int }}\right\rangle=-\int j_{\mu}(\mathbf{r}, t) A_{\mu}(\mathbf{r}, t) d^{3} \mathbf{r}, \quad\left\{\begin{array}{l}
j_{\mu}=(\mathbf{j}, i c \rho) \\
A_{\mu}=\left(\mathbf{A}, \frac{i}{c} \phi\right),
\end{array}\right.
$$

( $c$ is the speed of light) thus manifestly demonstrating its relativistic nature. In fact, one may very well argue that in the non-relativistic limit, electrodynamics reduces to electrostatics and that magnetic induction, in addition to retardation, is a relativistic effect. ${ }^{35}$ Yet, the minimal substitution is customarily employed also in calculations denoted as "non-relativistic." Such calculations in reality use a non-relativistic description of particles, but a relativistic treatment of their coupling to external electromagnetic fields. This is perfectly justified from a pragmatic point of view, but it should be kept in mind that if the sources of the electromagnetic waves were to be included in the system under study, their magnetic component would vanish.

A point we would like to emphasize in the present work is that the non-relativistic use of the minimal substitution in Eq. (1) leads to a more complicated formalism than the fully relativistic approach, since the former mixes theories of different transformation properties. This can be seen by comparing the non-relativistic and relativistic Hamiltonian operators obtained by minimal substitution. We may write the non-relativistic free-electron Hamiltonian in two different forms,

$$
\hat{h}_{0}^{N R}=\frac{\hat{p}^{2}}{2 m_{e}}=\frac{(\boldsymbol{\sigma} \cdot \hat{\mathbf{p}})^{2}}{2 m_{e}},
$$

where $m_{e}$ is the electron mass and $\sigma$ are the Pauli spin matrices. These two forms are equivalent as long as external fields are not invoked. Upon minimal substitution, one obtains

$$
\hat{h}^{N R}=\frac{\hat{p}^{2}}{2 m_{e}}+\frac{e}{2 m_{e}}(\mathbf{p} \cdot \mathbf{A}+\mathbf{A} \cdot \mathbf{p})+\frac{e^{2} A^{2}}{2 m_{e}}-e \phi+\frac{e \hbar}{2 m_{e}}(\boldsymbol{\sigma} \cdot \mathbf{B}),
$$

where $-e$ is the electron charge and $\hbar$ is the reduced Planck constant. The final term in Eq. (5), representing the spin-Zeeman interaction, only appears if one starts from the second form of Eq. (4). Spin can be thought of as hidden in the non-relativistic free-electron Hamiltonian. On the other hand, the first form of Eq. (4) can be thought of as a manifestation of the economy of Nature's laws: neither charge nor spin is required for the description of the free electron. We may contrast the non-relativistic Hamiltonian [Eq. (5)] with its relativistic counterpart,

$$
\hat{h}^{R}=\beta m_{e} c^{2}+c(\boldsymbol{\alpha} \cdot \hat{\mathbf{p}})+e c(\boldsymbol{\alpha} \cdot \mathbf{A})-e \phi,
$$

where $\boldsymbol{\alpha}$ and $\beta$ are the Dirac matrices. Here, the three terms describing magnetic interaction in the non-relativistic framework have been reduced to a single one, which is linear in the vector potential.

\section{B. Full light-matter interaction}

The Beer-Lambert law,

$$
I=I_{0} e^{-\mathcal{N} \sigma l},
$$

expresses the attenuation of the intensity $I_{0}$ of incoming light in terms of the effective number of absorbing molecules, given as the product of the number density $\mathcal{N}$ of absorbing molecules, the length $l$ of the sample, and the absorption cross section $\sigma$. To find an expression for the absorption cross section, we start from two equivalent expressions for the rate of energy exchange between (monochromatic) light and molecules: (i) as intensity times absorption cross section $\sigma$ or (ii) as photon energy $\hbar \omega$ times the transition rate $w_{f \leftarrow i}$, that is,

$$
I(\omega) \sigma(\omega)=\hbar \omega w_{f \leftarrow i}(\omega) .
$$

The intensity is expressed in terms of the electric constant $\varepsilon_{0}$ and the electric field strength $E_{\omega}$,

$$
I(\omega)=\frac{1}{2} \varepsilon_{0} c E_{\omega}^{2}
$$

Starting from a time-dependent interaction operator of the form

$$
\hat{V}(t)=\hat{V}(\omega) e^{-i \omega t}+\hat{V}(-\omega) e^{+i \omega t} ; \quad \hat{V}(-\omega)=\hat{V}^{\dagger}(\omega),
$$

an expression for the transition rate $w_{f \leftarrow i}$ may be found from timedependent perturbation theory, ${ }^{36}$

$$
w_{f \leftarrow i}(\omega)=\frac{2 \pi}{\hbar^{2}}\left|\left\langle f\left|\hat{V}\left(\omega_{f i}\right)\right| i\right\rangle\right|^{2} f\left(\omega, \omega_{f i}, \gamma_{f i}\right) .
$$

This formula is often referred to as Fermi's golden rule. However, the rule actually pertains to the transition from a discrete state to a continuum of states (see Ref. 37) but may be applied to a discrete final state, provided that it has a finite lifetime, ${ }^{38}$ here manifested by the line shape function $f\left(\omega, \omega_{f i}, \gamma_{f i}\right)$. Setting

$$
\hat{V}(\omega)=-\frac{1}{2} E_{\omega} \hat{T}(\omega)
$$

gives an expression for the absorption cross section,

$$
\sigma(\omega)=\frac{\pi \omega}{\varepsilon_{0} \hbar c}\left|\left\langle f\left|\hat{T}\left(\omega_{f i}\right)\right| i\right\rangle\right|^{2} f\left(\omega, \omega_{f i}, \gamma_{f i}\right)
$$

in terms of an effective interaction operator $\hat{T}(\omega)$ (see below). Closely related is the oscillator strength, defined as

$$
f_{f i}(\omega)=\frac{2 \omega}{\hbar e^{2}}\left|\left\langle f\left|\hat{T}\left(\omega_{f i}\right)\right| i\right\rangle\right|^{2} f\left(\omega, \omega_{f i}, \gamma_{f i}\right)
$$

In this work, we consider linearly polarized monochromatic light with electric and magnetic components,

$$
\begin{aligned}
& \mathbf{E}(\mathbf{r}, t)=E_{\omega} \boldsymbol{\epsilon} \sin [\mathbf{k} \cdot \mathbf{r}-\omega t+\delta], \\
& \mathbf{B}(\mathbf{r}, t)=\frac{E_{\omega}}{\omega}(\mathbf{k} \times \boldsymbol{\epsilon}) \sin [\mathbf{k} \cdot \mathbf{r}-\omega t+\delta],
\end{aligned}
$$


where $\mathbf{k}$ is the wave vector with length

$$
k=\frac{\omega}{c}=\frac{2 \pi}{\lambda},
$$

$\boldsymbol{\epsilon}$ is the polarization vector, and $\delta$ is the phase. Such an electromagnetic wave is conventionally represented in the Coulomb (radiation) gauge by the scalar and vector potentials,

$$
\tilde{\phi}(\mathbf{r}, t)=0, \quad \tilde{\mathbf{A}}(\mathbf{r}, t)=-\frac{E_{\omega}}{\omega} \boldsymbol{\epsilon} \cos [\mathbf{k} \cdot \mathbf{r}-\omega t+\delta] .
$$

Starting from the Dirac Hamiltonian in Eq. (6), this leads to an effective interaction operator of the form

$$
\hat{T}_{\text {full }}(\omega)=\frac{e}{\omega}(c \boldsymbol{\alpha} \cdot \boldsymbol{\epsilon}) e^{+i(\mathbf{k} \cdot \mathbf{r}+\delta)}, \quad \hat{T}_{\text {full }}^{\dagger}(\omega)=\hat{T}_{\text {full }}(-\omega) .
$$

It is clear from Eq. (5) that the corresponding effective interaction operator in the non-relativistic framework will have a more complicated expression. However, simplifications are introduced by invoking a weak-field approximation such that the third term, the diamagnetic contribution, is neglected. Also, the fifth term, the spin-Zeeman contribution, is often ignored.

One straightforwardly establishes that use of the full interaction operator assures gauge-origin independence of intensities. ${ }^{8}$ Upon a change of gauge-origin $\mathbf{O} \rightarrow \mathbf{O}+\mathbf{a}$, a constant complex phase is introduced in the interaction operator,

$$
\hat{T}_{\text {full }}(\omega ; \mathbf{O}) \rightarrow \hat{T}_{\text {full }}(\omega ; \mathbf{O}+\mathbf{a})=\hat{T}_{\text {full }}(\omega ; \mathbf{O}) e^{+i(\mathbf{k} \cdot \mathbf{a})} .
$$

This phase is, however, canceled by its complex conjugated partner when the interaction operator is inserted into the expressions for the absorption cross section [Eq. (13)] or oscillator strength [Eq. (14)].

The ED approximation assumes that the dimensionless quantity $\langle k r\rangle \ll 1$ such that the interaction operator may be approximated by

$$
\hat{T}_{V}(\omega)=\frac{e}{\omega}(c \boldsymbol{\alpha} \cdot \boldsymbol{\epsilon}) e^{+i \delta} \stackrel{\delta=0}{\longrightarrow} \quad \frac{e}{\omega}(c \boldsymbol{\alpha} \cdot \boldsymbol{\epsilon}),
$$

which physically corresponds to the absorbing molecule effectively seeing a uniform electric field. The subscript " $V$ " refers to the velocity representation. To convert to the length representation, we use the following expression for the velocity operator:

$$
\hat{\mathbf{v}}=-\frac{i}{\hbar}[\mathbf{r}, \hat{h}]
$$

obtained from the Heisenberg equation of motion, an observation that can be traced back at least to the first edition (1930) of Dirac's monograph. ${ }^{39}$ In the non-relativistic case, this leads to a velocity operator of the form $\hat{\mathbf{v}}^{\mathrm{NR}}=\hat{\mathbf{p}} / m_{e}$, which is straightforwardly related to the corresponding classical expression. In the relativistic case, one obtains the less intuitive form ${ }^{40,41} \hat{\mathbf{v}}^{\mathrm{R}}=c \boldsymbol{\alpha}$, expressing the Zitterbewegung of the electron, which facilitates the connection

$$
\begin{gathered}
\left\langle f\left|\hat{T}_{V}(\omega)\right| i\right\rangle=\left\langle f\left|\hat{T}_{L}(\omega)\right| i\right\rangle, \\
\hat{T}_{L}(\omega)=-i e^{+i \delta}\left(\frac{\omega_{f i}}{\omega}\right) \hat{\boldsymbol{\mu}} \cdot \boldsymbol{\epsilon} \quad \underset{\omega=\omega_{f i}}{\stackrel{\delta=\pi / 2}{\longrightarrow}} \hat{\boldsymbol{\mu}} \cdot \boldsymbol{\epsilon}, \quad \hat{\boldsymbol{\mu}}=-e \mathbf{r} .
\end{gathered}
$$

We prefer to refer to these forms as representations rather than gauges (see also Ref. 42 and references therein). Gauge freedom arises from the observation that the longitudinal component of the vector potential does not contribute to the magnetic field, and gauges are accordingly fixed by imposing conditions on this component. For instance, the condition $\nabla \cdot \mathbf{A}=0$ of the Coulomb gauge states that the longitudinal component of the vector potential is zero. Although the underlying potentials of the length and velocity representations are related by a gauge transformation, there is, as far as we can see, no gauge condition separating them. Both satisfy Coulomb gauge, but this is no longer the case for the length representation when going beyond the ED approximation, as demonstrated in Appendix B.

At this point, it should be noted that whereas the timedependent effective interaction operator $\hat{T}_{\text {full }}(t)$ is necessarily Hermitian, this is generally not the case for the frequency-dependent component $\hat{T}_{\text {full }}(\omega)$, as seen from Eq. (18). We shall, however, insist that the effective interaction operators are Hermitian within the ED approximation. This leads to the following choices for the phase $\delta$ of the electromagnetic plane wave [Eq. (15)],

$$
\delta= \begin{cases}0 & \text { (velocity representation) } \\ \pi / 2 & \text { (length representation) }\end{cases}
$$

In the present work, we report the implementation of three different schemes for the simulation of electronic spectra beyond the ED approximation within a linear response framework. More details about the underlying theory and the implementation are given in Appendix A. Two features of the present implementation of the full light-matter interaction operator should be stressed: (i) integrals over the effective interaction operator [Eq. (18)] in a Gaussian basis are identified as Fourier transforms with simple analytic expressions $^{8}$ and (ii) the effective interaction operator [Eq. (18)] is a general operator, and thus, it may be split into Hermitian and anti-Hermitian parts,

$$
\begin{aligned}
& \hat{T}_{H}(\omega)=\frac{e}{\omega}(c \boldsymbol{\alpha} \cdot \boldsymbol{\epsilon}) \cos (\mathbf{k} \cdot \mathbf{r}), \\
& \hat{T}_{A}(\omega)=\frac{e}{\omega}(i c \boldsymbol{\alpha} \cdot \boldsymbol{\epsilon}) \sin (\mathbf{k} \cdot \mathbf{r}) .
\end{aligned}
$$

The Hermitian and anti-Hermitian operators are time-antisymmetric and time-symmetric, respectively. In accordance with the quaternion symmetry scheme of DIRAC, ${ }^{43}$ an imaginary $i$ will be inserted in the Hermitian part to make it time-symmetric. The components can be further broken down on spatial symmetries using

$$
\begin{aligned}
& e^{ \pm i(\mathbf{k} \cdot \mathbf{r})}=\cos \left(k_{x} x\right) \cos \left(k_{y} y\right) \cos \left(k_{z} z\right) \quad\left(\Gamma_{0}\right) \\
& -\sin \left(k_{x} x\right) \sin \left(k_{y} y\right) \cos \left(k_{z} z\right) \quad\left(\Gamma_{R_{z}}\right) \\
& -\sin \left(k_{x} x\right) \cos \left(k_{y} y\right) \sin \left(k_{z} z\right) \quad\left(\Gamma_{R_{y}}\right) \\
& -\cos \left(k_{x} x\right) \sin \left(k_{y} y\right) \sin \left(k_{z} z\right) \quad\left(\Gamma_{R_{x}}\right) \\
& \mp i \sin \left(k_{x} x\right) \sin \left(k_{y} y\right) \sin \left(k_{z} z\right) \quad\left(\Gamma_{x y z}\right) \\
& \pm i \cos \left(k_{x} x\right) \cos \left(k_{y} y\right) \sin \left(k_{z} z\right)\left(\Gamma_{z}\right) \\
& \pm i \cos \left(k_{x} x\right) \sin \left(k_{y} y\right) \cos \left(k_{z} z\right)\left(\Gamma_{y}\right) \\
& \pm i \sin \left(k_{x} x\right) \cos \left(k_{y} y\right) \cos \left(k_{z} z\right)\left(\Gamma_{x}\right) \text {. }
\end{aligned}
$$

Here, $\Gamma_{0}$ refers to the totally symmetric irrep, $\left(\Gamma_{x}, \Gamma_{y}, \Gamma_{z}\right)$ refers to the symmetries of the coordinates, $\left(\Gamma_{R_{x}}, \Gamma_{R_{y}}, \Gamma_{R_{z}}\right)$ refers to the symmetry of the rotations, and $\Gamma_{x y z}$ refers to the symmetry of 
the function $x y z$. Together, these eight symmetries form the eight irreps of the $D_{2 h}$ point group, whereas some symmetries coalesce for subgroups. In the present implementation, for an excitation of given (boson) symmetry, we only invoke the relevant contribution from $e^{ \pm i(\mathbf{k} \cdot \mathbf{r})}$.

\section{Truncated light-matter interaction}

In this section, we derive expressions for the absorption cross section or oscillator strength truncated to finite order in the length of the wave vector. In the first subsection, we develop a compact formalism based directly on an expansion of the effective interaction operator [Eq. (18)]. Next, we provide the relativistic extension of the theory developed by Bernadotte and co-workers, ${ }^{17}$ where oscillator strengths are expressed in terms of electric and magnetic multipoles. We shall, however, obtain these expressions in a more straightforward manner by using the multipolar gauge. The two approaches can, to some extent, be thought of as generalizations of the velocity and length representations, respectively, to arbitrary orders in the wave vector

\section{Coulomb gauge: Velocity representation}

A direct approach for obtaining the absorption cross section (or oscillator strength) to some order in the wave vector is to perform a Taylor-expansion of the absorption cross section in Eq. (13) in orders of the length of the wave vector, that is,

$$
\begin{aligned}
\sigma(\omega) & =\frac{\pi \omega}{\varepsilon_{0} \hbar c} \sum_{n=0}^{\infty} \frac{k^{n}}{n !} \frac{d^{n}}{d k^{n}}\left[\left\langle f\left|\hat{T}_{\text {full }}\left(\omega_{f i}\right)\right| i\right\rangle\left\langle f\left|\hat{T}_{\text {full }}\left(\omega_{f i}\right)\right| i\right\rangle^{*}\right]_{k=0} f\left(\omega, \omega_{f i}, \gamma_{f i}\right) \\
& =\frac{\pi \omega}{\varepsilon_{0} \hbar c} \sum_{n=0}^{\infty} \sum_{m=0}^{n}\left\langle f\left|\hat{T}_{\text {full }}^{[n-m]}\left(\omega_{f i}\right)\right| i\right\rangle\left\langle f\left|\hat{T}_{\text {full }}^{[m]}\left(\omega_{f i}\right)\right| i\right\rangle^{*} f\left(\omega, \omega_{f i}, \gamma_{f i}\right),
\end{aligned}
$$

where Taylor coefficients

$$
\hat{T}_{\text {full }}^{[n]}(\omega)=\frac{k^{n}}{n !} \frac{d^{n}}{d k^{n}}\left[\frac{e}{\omega}(c \boldsymbol{\alpha} \cdot \boldsymbol{\epsilon}) e^{+i(\mathbf{k} \cdot \mathbf{r})}\right]_{k=0}=\frac{e}{\omega} \frac{1}{n !}(c \boldsymbol{\alpha} \cdot \boldsymbol{\epsilon})(i \mathbf{k} \cdot \mathbf{r})^{n}
$$

appear in the corresponding expansion of the effective interaction operator [Eq. (18)] with the phase $\delta=0$, according to the phase convention in Eq. (24). From inspection, we find that even- and odd-order operators are time-antisymmetric and time-symmetric, respectively. It should be noted that the underlying, truncated vector potential satisfies the Coulomb gauge.

We may separate the absorption cross section into even- and odd-order contributions with respect to the wave vector, that is,

$$
\begin{aligned}
\sigma^{[2 n]}(\omega)= & \frac{\pi \omega}{\varepsilon_{0} \hbar c} \sum_{m=0}^{n}\left(2-\delta_{m 0}\right) \operatorname{Re}\left\{\left\langle f\left|\hat{T}_{\text {full }}^{[n-m]}\left(\omega_{f i}\right)\right| i\right\rangle\left\langle f\left|\hat{T}_{\text {full }}^{[n+m]}\left(\omega_{f i}\right)\right| i\right\rangle^{*}\right\} \\
& \times f\left(\omega, \omega_{f i}, \gamma_{f i}\right), \\
\sigma^{[2 n+1]}(\omega)= & \frac{\pi \omega}{\varepsilon_{0} \hbar c} \sum_{m=0}^{n} 2 \operatorname{Re}\left\{\left\langle f\left|\hat{T}_{\text {full }}^{[n+m+1]}\left(\omega_{f i}\right)\right| i\right\rangle\left\langle f\left|\hat{T}_{\text {full }}^{[n-m]}\left(\omega_{f i}\right)\right| i\right\rangle^{*}\right\} \\
& \times f\left(\omega, \omega_{f i}, \gamma_{f i}\right)=0 .
\end{aligned}
$$

The odd-order contributions vanish identically because the two interaction operators of each term, contrary to the even-order terms, will have opposite symmetry with respect to time reversal such that the product of their transition moments will be imaginary (see Appendix A).

The demonstration of formal gauge-origin independence in the generalized velocity representation at each order $n$ in the wave vector follows straightforwardly from Eq. (28), being $n$th order derivatives of a term that is gauge-origin independent for all values of $k$. This result was obtained earlier in the non-relativistic framework by Bernadotte et al. but in a somewhat elaborate manner (see Appendix C of Ref. 17). Their derivation, however, highlights the challenge of achieving gauge-origin independence in practical calculations, and so, we shall give a slightly more compact version here: Upon a change of gauge-origin $\mathbf{O} \rightarrow \mathbf{O}+\mathbf{a}$, the $n$th order interaction operator in the velocity representation may be expressed as

$\hat{T}_{\text {full }}^{[n]}(\omega ; \mathbf{O}) \rightarrow \hat{T}_{\text {full }}^{[n]}(\omega ; \mathbf{O}+\mathbf{a})=\sum_{m=0}^{n} \frac{1}{m !}(i \mathbf{k} \cdot \mathbf{a})^{m} \hat{T}_{\text {full }}^{[n-m]}(\omega ; \mathbf{O})$,

which follows from Eq. (19). The $n$th order absorption cross section at the new gauge origin may then be expressed as

$$
\begin{aligned}
\sigma^{[n]}(\omega ; \mathbf{O}+\mathbf{a})= & \sum_{m=0}^{n}\left\langle f\left|T_{\text {full }}^{[n-m]}(\omega ; \mathbf{O}+\mathbf{a})\right| i\right\rangle\left\langle f\left|T_{\text {full }}^{[m]}(\omega ; \mathbf{O}+\mathbf{a})\right| i\right\rangle^{*} \\
= & \sum_{m=0}^{n} \sum_{p=0}^{n-m} \sum_{q=0}^{m} \frac{1}{p ! q !}(-1)^{q}(i \mathbf{k} \cdot \mathbf{a})^{p+q} \\
& \times\left\langle f\left|T_{\text {full }}^{[n-m-p]}(\omega ; \mathbf{O})\right| i\right\rangle\left\langle f\left|T_{\text {full }}^{[m-q]}(\omega ; \mathbf{O})\right| i\right\rangle^{*} .
\end{aligned}
$$

If we take the orders for each pair of interaction operators as indices of a matrix, we see that the pairs $(n-m, m)$ from the first line fill the antidiagonal of a square matrix with indices running from 0 to $n$, whereas the pairs $(n-m-p, n-q)$ from the second line fill the triangle above as well. This suggests to replace indices $m$ and $p$ by $u=n-m-p$ and $v=m-q$. After rearrangement, this leads to the expression

$$
\begin{aligned}
\sigma^{[n]}(\omega ; \mathbf{O}+\mathbf{a})= & \sum_{v=0}^{n} \sum_{u=0}^{n-v}\left\langle f\left|T_{\text {full }}^{[u]}(\omega ; \mathbf{O})\right| i\right\rangle\left\langle f\left|T_{\text {full }}^{[v]}(\omega ; \mathbf{O})\right| i\right\rangle^{*} \\
& \times \frac{(\mathbf{k} \cdot \mathbf{a})^{n-(u+v)}}{(n-(u+v)) !} \times \mathcal{M},
\end{aligned}
$$

where

$$
\mathcal{M}=\sum_{q=0}^{n-(u+v)}\left(\begin{array}{c}
(n-(u+v)) \\
q
\end{array}\right)(-1)^{q}=(1-1)^{n-(u+v)} .
$$

The factor $\mathcal{M}$ is zero unless $u=n-v$, which reduces the second line of Eq. (33) to the same form as the first line, hence demonstrating that $\sigma^{[n]}(\omega ; \mathbf{O}+\mathbf{a})=\sigma^{[n]}(\omega ; \mathbf{O})$, that is, the oscillator strengths are indeed gauge-origin independent. However, one should note that the lower-order interaction operators introduced in Eq. (32) upon a change of gauge origin involve multiplication with powers of the displacement as well as the wave vector. This may eventually introduce numerical issues, as will be shown in Sec. IV A 2.

\section{Multipolar gauge: Length representation}

A very convenient way of introducing electric and magnetic multipoles is through the use of multipolar gauge, ${ }^{36,44-46}$ also known 
as Bloch gauge, ${ }^{47,48}$ Barron-Gray gauge, ${ }^{18}$ or Poincaré gauge, ${ }^{49-52}$ reflecting a history of multiple rediscoveries. In Appendix B, we provide a compact derivation of the multipolar gauge, avoiding excessive use of indices. In multipolar gauge, the potentials are given in terms of the electric and magnetic fields and their derivatives at some expansion point a. When inserted into the interaction Hamiltonian in Eq. (2), they automatically provide an expansion of the lightmatter interaction in terms of electric and magnetic multipoles of the molecule.

We first consider the form of the effective interaction in the multipolar gauge, starting from the electromagnetic plane wave, Eq. (15), represented by the potentials in Eq. (17). In accordance with the discussion in Sec. II B and the phase convention of Eq. (24), we set the phase of the plane wave to $\delta=\pi / 2$. The potentials in the multipolar gauge (mg) are then given by

$$
\begin{aligned}
\phi(\mathbf{r}, t)= & -\frac{1}{2}\left(\boldsymbol{\delta} \cdot \mathbf{E}_{\omega}\right) \sum_{n=0}^{\infty} \frac{1}{(n+1) !}\left\{(i \mathbf{k} \cdot \boldsymbol{\delta})^{n} e^{i(\mathbf{k} \cdot \mathbf{a}-\omega t)}\right. \\
& \left.+(-i \mathbf{k} \cdot \boldsymbol{\delta})^{n} e^{-i(\mathbf{k} \cdot \mathbf{a}-\omega t)}\right\}, \quad \boldsymbol{\delta}=\mathbf{r}-\mathbf{a}, \\
\mathbf{A}(\mathbf{r}, t)= & -\frac{1}{2}\left(\boldsymbol{\delta} \times \mathbf{B}_{\omega}\right) \sum_{n=1}^{\infty} \frac{n}{(n+1) !}\left\{(i \mathbf{k} \cdot \boldsymbol{\delta})^{n-1} e^{i(\mathbf{k} \cdot \mathbf{a}-\omega t)}\right. \\
& \left.+(-i \mathbf{k} \cdot \boldsymbol{\delta})^{n-1} e^{-i(\mathbf{k} \cdot \mathbf{a}-\omega t)}\right\} .
\end{aligned}
$$

Setting the expansion point $\mathbf{a}=\mathbf{0}$, we find that the effective interaction operator may be expressed as

$$
\hat{T}_{\mathrm{mg}}=\sum_{n=0}^{\infty} \hat{T}_{\mathrm{mg}}^{[n]},
$$

where

$$
\begin{gathered}
\hat{T}_{\mathrm{mg}}^{[0]}(\omega)=-e(\mathbf{r} \cdot \boldsymbol{\epsilon}), \\
\hat{T}_{\mathrm{mg}}^{[n]}(\omega)=-e\left[\frac{1}{(n+1) !}(\mathbf{r} \cdot \boldsymbol{\epsilon})(i \mathbf{k} \cdot \mathbf{r})^{n}-\frac{i}{\omega} \frac{n}{(n+1) !}\right. \\
\left.\times(i \mathbf{k} \times \boldsymbol{\epsilon}) \cdot(\mathbf{r} \times c \boldsymbol{\alpha})(i \mathbf{k} \cdot \mathbf{r})^{n-1}\right], \quad n \neq 0 .
\end{gathered}
$$

Further insight is obtained by writing the effective interaction operator on component form as

$$
\hat{T}_{\mathrm{mg}}(\omega)=\hat{Q}_{p}^{[1]} \epsilon_{p}+\sum_{n=1}^{\infty} i^{n} \epsilon_{p} k_{j_{1}} k_{j_{2}} \ldots k_{j_{n}} \hat{X}_{j_{1} \ldots j_{n} ; p}^{[n]}(\omega) .
$$

In the above expression, we employ the Einstein summation convention and introduce the multipole operator $\hat{X}^{[n]}$ associated with $O\left(k^{n}\right)$,

$$
\hat{X}_{j_{1} \ldots j_{n} ; p}^{[n]}(\omega)=\frac{1}{(n+1) !} \hat{Q}_{j_{1} \ldots j_{n}, p}^{[n+1]}-\frac{i}{\omega} \frac{1}{n !} \hat{m}_{j_{1} \ldots j_{n-1} ; r}^{[n]} \varepsilon_{r j_{n} p},
$$

where $\varepsilon_{i j k}$ is the Levi-Civita symbol. This operator is in turn built from the electric and magnetic multipole operators,

$$
\begin{gathered}
\hat{Q}_{j_{1} \ldots j_{n}}^{[n]}=-e r_{j_{1}} r_{j_{2}} \ldots r_{j_{n}}, \\
\hat{m}_{j_{1} \ldots j_{n-1} ; j_{n}}^{[n]}=\frac{n}{n+1} r_{j_{1}} r_{j_{2}} \ldots r_{j_{n-1}}(\mathbf{r} \times \hat{\mathbf{j}})_{j_{n}}, \quad \hat{\mathbf{j}}=-e c \boldsymbol{\alpha} .
\end{gathered}
$$

Again, we would like to stress the simplicity of the relativistic formalism compared to the non-relativistic one: the magnetic multipole operators $\hat{m}^{[n]}$ contain the current density operator $\hat{\mathbf{j}}$, which in the relativistic form is simply electron charge times the velocity operator, allowing straightforward implementation of the magnetic multipole operator to arbitrary orders. The non-relativistic form is more involved, containing contributions from the mechanical momentum operator as well as the curl of the spin magnetization. ${ }^{53}$ One may note that the electric and magnetic multipole operators are time-symmetric and time-antisymmetric, respectively. However, in Eq. (42), the magnetic multipole operator is multiplied with imaginary $i$ such that the multipole operator $\hat{X}^{[n]}$ is time-symmetric, fitting well into the quaternion symmetry scheme of DIRAC.

Inserting the effective interaction operator $\hat{T}_{\mathrm{mg}}(\omega)$ into the expression for the absorption cross section in Eq. (13) and expanding in orders of the wave vector, we find that odd-order contributions to the absorption cross section vanish, as was also the case in the velocity representation, whereas the even-order ones may be expressed as

$$
\begin{aligned}
\sigma^{[2 n]}(\omega)= & \frac{\pi \omega}{\varepsilon_{0} \hbar c} \sum_{m=0}^{n}(-1)^{m}\left(2-\delta_{m 0}\right) \epsilon_{p} \epsilon_{q} k_{j_{1}} k_{j_{2}} \ldots k_{j_{2 n}} \\
& \times \operatorname{Re}\left\{\left\langle f\left|\hat{X}_{j_{1} \ldots j_{n+m} ; p}^{[n+m]}(\omega)\right| i\right\rangle\left\langle f\left|\hat{X}_{j_{n+m+1} \ldots j_{2 n} ; q}^{[n-m]}(\omega)\right| i\right\rangle^{*}\right\} \\
& \times f\left(\omega, \omega_{f i}, \gamma_{f i}\right) .
\end{aligned}
$$

We may connect the interaction operators of multipolar gauge with those of the Coulomb gauge in the velocity representation. Starting from Eq. (29), we use the relation

$$
(i \mathbf{k} \times \boldsymbol{\epsilon}) \cdot(\mathbf{r} \times c \boldsymbol{\alpha})=(c \boldsymbol{\alpha} \cdot \boldsymbol{\epsilon})(i \mathbf{k} \cdot \mathbf{r})-(\mathbf{r} \cdot \boldsymbol{\epsilon})(i \mathbf{k} \cdot c \boldsymbol{\alpha})
$$

to obtain

$$
\begin{aligned}
T^{[n]}(\omega)= & \frac{e}{\omega} \frac{1}{(n+1) !}\left\{(c \boldsymbol{\alpha} \cdot \boldsymbol{\epsilon})(i \mathbf{k} \cdot \mathbf{r})^{n}+n(\mathbf{r} \cdot \boldsymbol{\epsilon})(i \mathbf{k} \cdot c \boldsymbol{\alpha})(i \mathbf{k} \cdot \mathbf{r})^{n-1}\right\} \\
& +\frac{e}{\omega} \frac{n}{(n+1) !}(i \mathbf{k} \times \boldsymbol{\epsilon}) \cdot(\mathbf{r} \times c \boldsymbol{\alpha})(i \mathbf{k} \cdot \mathbf{r})^{n-1} .
\end{aligned}
$$

Comparing with Eq. (40), we see that the second term above contains the $n$ th-order magnetic multipole operator, which implies that we may extract from the first term the $(n+1)$ th-order electric multipole operator in the velocity representation. Next, we use

$$
-\frac{i}{\hbar}\left[(\mathbf{r} \cdot \boldsymbol{\epsilon})(i \mathbf{k} \cdot \mathbf{r})^{n}, \hat{h}\right]=(c \boldsymbol{\alpha} \cdot \boldsymbol{\epsilon})(i \mathbf{k} \cdot \mathbf{r})^{n}+n(\mathbf{r} \cdot \boldsymbol{\epsilon})(i \mathbf{k} \cdot c \boldsymbol{\alpha})(i \mathbf{k} \cdot \mathbf{r})^{n-1}
$$

to arrive at

$$
\begin{aligned}
T^{[n]}(\omega)= & \frac{-i e}{\hbar \omega} \frac{1}{(n+1) !}\left[(\mathbf{r} \cdot \boldsymbol{\epsilon})(i \mathbf{k} \cdot \mathbf{r})^{n}, \hat{h}\right] \\
& +\frac{e}{\omega} \frac{n}{(n+1) !}(i \mathbf{k} \times \boldsymbol{\epsilon}) \cdot(\mathbf{r} \times c \boldsymbol{\alpha})(i \mathbf{k} \cdot \mathbf{r})^{n-1} .
\end{aligned}
$$

In order to complete the derivation, we have to form transition moments, which provide the connection

$$
\left\langle f\left|\hat{T}_{\text {full }}^{[n]}(\omega)\right| i\right\rangle=-i\left\langle f\left|\hat{T}_{\mathrm{mg}}^{[n]}(\omega)\right| i\right\rangle
$$

between velocity and length representations and generalize Eq. (22) to arbitrary orders in the wave vector; in Eq. (22), the negative imaginary phase is canceled by choosing the phase $\delta=\pi / 2$, which is 
not done here. At this point, one should note that the derivation is greatly simplified by the fact that the relativistic velocity operator $c \boldsymbol{\alpha}$ commutes with the coordinates, contrary to the non-relativistic one. Furthermore, as discussed at the end of Appendix B, the appearance of a commutator involving the Hamiltonian can be taken as an indication of a gauge transformation, and indeed, we show that the operator appearing together with the Hamiltonian in Eq. (48) is the gauge function of the multipolar gauge [Eq. (B4)] obtained by inserting the vector potential [Eq. (17)] of a linear plane wave and retaining the term of order $n$ in the wave vector.

The multipolar gauge has mostly been discussed in the framework of atomic physics where the nuclear origin provides a natural expansion point. In a molecule, there is generally no natural expansion point, and gauge-origin independence becomes an issue. Starting from Eq. (32) and using the connection Eq. (50), one straightforwardly derives

$$
\begin{aligned}
& \left\langle f\left|\hat{T}_{\mathrm{mg}}^{[n]}(\omega ; \mathbf{O})\right| i\right\rangle \rightarrow\left\langle f\left|\hat{T}_{\mathrm{mg}}^{[n]}(\omega ; \mathbf{O}+\mathbf{a})\right| i\right\rangle \\
& \quad=\sum_{m=0}^{n} \frac{1}{m !}(i \mathbf{k} \cdot \mathbf{a})^{m}\left\langle f\left|\hat{T}_{\mathrm{mg}}^{[n-m]}(\omega ; \mathbf{O})\right| i\right\rangle,
\end{aligned}
$$

from which gauge-origin independence of absorption cross sections to all orders in the wave vector follows using the same demonstration as for the Coulomb gauge (velocity representation) in Sec. II C 1. However, the demonstration this time hinges on the connection Eq. (50), which is established using commutator relations involving the Hamiltonian that do not necessarily hold in a finite basis and which effectively amounts to a gauge transformation. We have not been able to show gauge-origin independence of absorption cross sections while staying within the multipolar gauge, except for the zeroth order term (electric-dipole approximation) where it follows from orthogonality of states. In fact, in Appendix B, we show that potentials derived with respect to two different expansion points are related by a gauge transformation, but apparently only to the extent that the expansion is not truncated. This suggests that the lack of origin invariance of oscillator strengths observed by Bernadotte et al. ${ }^{17}$ and others, including us (see below), in calculations using an effective interaction operator on multipolar form is more than a finite basis set effect. It also makes sense since truncating the Taylor expansion of electric and magnetic fields inevitably conserves only local information.

\section{Rotational averages}

\section{General}

An often encountered experimental situation involves freely rotating molecules, and we will therefore have to consider rotational averaging. However, rather than rotating the molecules, we shall rotate the experimental configuration. To this end, we use the unit vectors of the spherical coordinates,

$$
\begin{aligned}
\mathbf{e}_{r} & =\mathbf{e}_{x} \sin \theta \cos \phi+\mathbf{e}_{y} \sin \theta \sin \phi+\mathbf{e}_{z} \cos \theta, \\
\mathbf{e}_{\theta} & =\mathbf{e}_{x} \cos \theta \cos \phi+\mathbf{e}_{y} \cos \theta \sin \phi-\mathbf{e}_{z} \sin \theta, \\
\mathbf{e}_{\phi} & =-\mathbf{e}_{x} \sin \phi+\mathbf{e}_{y} \cos \phi,
\end{aligned}
$$

which reduce to $\left(\mathbf{e}_{z}, \mathbf{e}_{x}, \mathbf{e}_{y}\right)$ when the angles $\theta$ and $\phi$ are both set to zero. More precisely, we shall align the wave unit vector $\mathbf{e}_{k}$ with the radial unit vector $\mathbf{e}_{r}$. The polarization vector $\boldsymbol{\epsilon}$ is then in the plane spanned by the unit vectors $\mathbf{e}_{\theta}$ and $\mathbf{e}_{\phi}$. Accordingly, we set

$$
\mathbf{e}_{k}=\mathbf{e}_{r}, \quad \boldsymbol{\epsilon}=\cos \chi \mathbf{e}_{\theta}+\sin \chi \mathbf{e}_{\phi},
$$

introducing a third angle $\chi$. The rotational average is defined as

$$
\langle g(\mathbf{r})\rangle_{\theta, \phi, \chi}=\frac{1}{8 \pi^{2}} \int_{0}^{2 \pi} \int_{0}^{2 \pi} \int_{0}^{\pi} g(\mathbf{r}) \sin \theta d \theta d \phi d \chi .
$$

\section{Full light-matter interaction}

Starting from Eq. (13), the rotationally averaged absorption cross section reads (for any choice of the phase $\delta$ )

$$
\begin{aligned}
\langle\sigma(\omega)\rangle_{\theta, \phi, \chi}= & \frac{\pi \omega}{\varepsilon_{0} \hbar c}\left(\frac{e}{\omega_{f i}}\right)^{2}\left\langle\epsilon_{p} \epsilon_{q}\left\langle f\left|c \alpha_{p} e^{+i(\mathbf{k} \cdot \mathbf{r})}\right| i\right\rangle\right. \\
& \left.\times\left\langle f\left|c \alpha_{q} e^{+i(\mathbf{k} \cdot \mathbf{r})}\right| i\right\rangle^{*}\right\rangle_{\theta, \phi, \chi} f\left(\omega, \omega_{f i}, \gamma_{f i}\right) .
\end{aligned}
$$

We first note that the $\chi$-dependence only enters the polarization vector $\boldsymbol{\epsilon}$ so that we may write

$$
\begin{aligned}
\langle\sigma(\omega)\rangle_{\theta, \phi, \chi}= & \frac{\pi \omega}{\varepsilon_{0} \hbar c}\left(\frac{e}{\omega_{f i}}\right)^{2}\left\langle\left\langle\epsilon_{p} \epsilon_{q}\right\rangle_{\chi}\left\langle f\left|c \alpha_{p} e^{+i(\mathbf{k} \cdot \mathbf{r})}\right| i\right\rangle\right. \\
& \left.\times\left\langle f\left|c \alpha_{q} e^{+i(\mathbf{k} \cdot \mathbf{r})}\right| i\right\rangle^{*}\right\rangle_{\theta, \phi} f\left(\omega, \omega_{f i}, \gamma_{f i}\right) .
\end{aligned}
$$

The $\chi$-average has a simple analytic expression in terms of the components of the radial unit vector,

$$
\left\langle\epsilon_{p} \epsilon_{q}\right\rangle_{\chi}=\frac{1}{2}\left(e_{\theta ; p} e_{\theta ; q}+e_{\phi ; p} e_{\phi ; q}\right)=\frac{1}{2}\left(\delta_{p q}-e_{r ; p} e_{r ; q}\right)
$$

which follows from the orthonormality of the unit vectors [Eq. (52)]. The $(\theta, \phi)$-average, on the other hand, will be handled numerically using Lebedev quadrature, ${ }^{54-59}$ which we, in our corresponding nonrelativistic work, have found to converge quickly. ${ }^{25}$

\section{Truncated light-matter interaction}

In the generalized velocity representation of Sec. II C 1 , the rotational average initially reads

$$
\begin{aligned}
\left\langle\sigma^{[2 n]}(\omega)\right\rangle_{\theta, \phi, \chi}= & \frac{\pi \omega}{\varepsilon_{0} \hbar c}\left(\frac{e}{\omega_{f i}}\right)^{2} \sum_{m=0}^{n} \frac{(-1)^{m}}{(n+m) !(n-m) !}\left(2-\delta_{m 0}\right) \\
& \times\left(\frac{\omega_{f i}}{c}\right)^{2 n}\left\langle\left\langle\epsilon_{p} \epsilon_{q}\right\rangle_{\chi} e_{r ; j_{1}} e_{r ; j_{2}} \ldots e_{r ; j_{2 n}}\right\rangle_{\theta, \phi} \\
& \times \operatorname{Re}\left\{\left\langle f\left|i c \alpha_{p} r_{j_{1}} \ldots r_{j_{n+m}}\right| i\right\rangle\left\langle f\left|i c \alpha_{q} r_{j_{n+m+1}} \ldots r_{j_{2 n}}\right| i\right\rangle^{*}\right\} \\
& \times f\left(\omega, \omega_{f}, \gamma_{f i}\right),
\end{aligned}
$$

whereas in the generalized length representation (multipolar gauge), the corresponding expression is

$$
\begin{aligned}
\left\langle\sigma^{[2 n]}(\omega)\right\rangle_{\theta, \phi, \chi}= & \frac{\pi \omega}{\varepsilon_{0} \hbar c} \sum_{m=0}^{n}(-1)^{m}\left(2-\delta_{m 0}\right)\left(\frac{\omega_{f i}}{c}\right)^{2 n} \\
& \times\left\langle\left\langle\epsilon_{p} \epsilon_{q}\right\rangle_{\chi} e_{r ; j_{1}} e_{r ; j_{2}} \ldots e_{r ; j_{2 n}}\right\rangle_{\theta, \phi} \\
& \times \operatorname{Re}\left\{\left\langle f\left|\hat{X}_{j_{1} \ldots j_{n+m} ; p}^{[n+m]}(\omega)\right| i\right\rangle\left\langle f\left|\hat{X}_{j_{n+m+1} \ldots j_{2 n} ; q}^{[n-m]}(\omega)\right| i\right\rangle^{*}\right\} \\
& \times f\left(\omega, \omega_{f i}, \gamma_{f i}\right) .
\end{aligned}
$$


In both cases, the central quantity to evaluate is

$$
\begin{aligned}
& \left\langle\left\langle\epsilon_{p} \epsilon_{q}\right\rangle_{\chi} e_{r ; j_{1}} e_{r ; j_{2}} \ldots e_{r ; j_{2 n}}\right\rangle_{\theta, \phi} \\
& =\frac{1}{4 \pi} \int_{0}^{2 \pi} \int_{0}^{\pi}\left\langle\epsilon_{p} \epsilon_{q}\right\rangle_{\chi} e_{r ; j_{1}} e_{r ; j_{2}} \ldots e_{r ; j_{2 n}} \sin \theta d \theta d \phi .
\end{aligned}
$$

Since the integrand is fully symmetric in indices $\left(j_{1}, \ldots j_{2 n}\right)$, we can collect contributions to the three components of the wave unit vector to give

$$
\begin{gathered}
\left\langle\left\langle\epsilon_{p} \epsilon_{q}\right\rangle_{\chi} e_{r ; j_{1}} e_{r ; j_{2}} \ldots e_{r ; j_{2 n}}\right\rangle_{\theta, \phi} \\
=\frac{1}{8 \pi} \int_{0}^{2 \pi} \int_{0}^{\pi}\left(\delta_{p q}-e_{r ; p} e_{r ; q}\right) e_{r ; x}^{i} e_{r ; y}^{j} e_{r ; z}^{k} \sin \theta d \theta d \phi ; \\
i+j+k=2 n .
\end{gathered}
$$

The calculation of the rotational averages thus hinges on the evaluation of expressions of the form

$$
\begin{aligned}
E_{t u v} & =\frac{1}{8 \pi} \int_{0}^{2 \pi} \int_{0}^{\pi} e_{r ; x}^{t} e_{r ; y}^{u} e_{r ; z}^{v} \sin \theta d \theta d \phi \\
& =\frac{1}{8 \pi} \int_{0}^{2 \pi} \cos ^{t} \phi \sin ^{u} \phi \int_{0}^{\pi} \cos ^{v} \theta \sin ^{t+u+1} \theta d \theta d \phi .
\end{aligned}
$$

A computational useful expression is obtained in two steps. First, we use the relations

$$
\begin{gathered}
\int_{0}^{\pi} \cos ^{p} \theta \sin ^{q} \theta d \theta=\left[1+(-1)^{p}\right] \int_{0}^{\pi / 2} \cos ^{p} \theta \sin ^{q} \theta d \theta \\
\int_{0}^{2 \pi} \cos ^{p} \phi \sin ^{q} \phi d \phi=\left[1+(-1)^{p}\right]\left[1+(-1)^{q}\right] \int_{0}^{\pi / 2} \cos ^{p} \phi \sin ^{q} d \phi
\end{gathered}
$$

to reduce the angular integration to the $(+,+,+)$ octant of Euclidean space,

$$
\begin{aligned}
E_{t u v}= & \frac{1}{8 \pi}\left[1+(-1)^{t}\right]\left[1+(-1)^{u}\right]\left[1+(-1)^{v}\right] \\
& \times \int_{0}^{\pi / 2} \int_{0}^{\pi / 2} e_{r ; x}^{t} e_{r ; y}^{u} e_{r ; z}^{v} \sin \theta d \theta d \phi
\end{aligned}
$$

This provides a powerful selection rule, showing that the expression $E_{t u v}$ is zero unless all integer exponents $t, u$, and $v$ are even. In passing, we note that the selection rule is the same for both terms appearing in Eq. (61) for $p=q$. Second, we use the integral representation (see Appendix C),

$$
\begin{aligned}
B(a, b, c) & =\frac{\Gamma(a) \Gamma(b) \Gamma(c)}{\Gamma(a+b+c)} \\
& =4 \int_{0}^{\pi / 2} \int_{0}^{\pi / 2} e_{r ; x}^{2 a-1} e_{r ; y}^{2 b-1} e_{r ; z}^{2 c-1} \sin \theta d \theta d \phi,
\end{aligned}
$$

to express the rotational average in terms of the trivariate beta function $B(a, b, c)$,

$$
\begin{aligned}
E_{\text {tuv }}= & \frac{1}{32 \pi}\left[1+(-1)^{t}\right]\left[1+(-1)^{u}\right]\left[1+(-1)^{v}\right] \\
& \times B\left(\frac{t+1}{2}, \frac{u+1}{2}, \frac{v+1}{2}\right) .
\end{aligned}
$$

The final result is thereby

$$
E_{\text {tuv }}= \begin{cases}\frac{(t-1) !(u-1) ! !(v-1) ! !}{2(t+u+v+1) ! !}, & t, u, v \text { even } \\ 0, & \text { otherwise }\end{cases}
$$

where we have used the identity

$$
\Gamma\left(\frac{t+1}{2}\right)=(t-1) ! ! \sqrt{\frac{\pi}{2^{t}}}
$$

for the evaluation of the trivariate beta function.

Our approach is different from the conventional approach to rotational averages using linear combinations of fundamental Cartesian isotropic tensors. ${ }^{60-65}$ The fundamental Cartesian isotropic tensors of even rank are given by products of Kronecker deltas $\delta_{i j}$, whereas an additional Levi-Civita symbol $\epsilon_{i j k}$ appears at odd rank. ${ }^{66-68}$ For instance, connecting to the notation of Barron, ${ }^{19}$ the rotational average appearing in the second-order contribution $\sigma^{[2]}$ to the absorption cross section is

$$
\left\langle\left\langle\epsilon_{\alpha} \epsilon_{\beta}\right\rangle_{\chi} e_{r ; \gamma} e_{r ; \delta}\right\rangle_{\theta, \phi}=\left\langle i_{\alpha} i_{\beta} k_{\gamma} k_{\delta}\right\rangle=\frac{1}{30}\left(4 \delta_{\alpha \beta} \delta_{\gamma \delta}-\delta_{\alpha \gamma} \delta_{\beta \delta}-\delta_{\alpha \delta} \delta_{\beta \delta}\right) .
$$

The established procedure for generating a suitable linearly independent set of fundamental Cartesian isotropic tensors involves the construction of standard tableaux from Young diagrams. ${ }^{61,69}$ For even rank, one can connect to our approach from the observation that the integer exponents $t, u$, and $v$ in the expression for $E_{t u v}$ [Eq. (67)] must all be even. This implies a pairing of indices, which can be expressed through strings of Kronecker deltas. Simple combinatorics suggests that the possible number of pairings of $2 n$ indices and thus the number of fundamental Cartesian isotropic tensors of even rank $2 n$ are $(2 n-1)$ !!. However, starting at rank eight, linear dependencies (syzygies) occur, ${ }^{60,62,70}$ e.g.,

$$
\left|\begin{array}{llll}
\delta_{i_{1} i_{5}} & \delta_{i_{1} i_{6}} & \delta_{i_{1} i_{7}} & \delta_{i_{1} i_{8}} \\
\delta_{i_{2} i_{5}} & \delta_{i_{2} i_{6}} & \delta_{i_{2} i_{7}} & \delta_{i_{2} i_{8}} \\
\delta_{i_{3} i_{5}} & \delta_{i_{3} i_{6}} & \delta_{i_{3} i_{7}} & \delta_{i_{3} i_{8}} \\
\delta_{i_{4} i_{5}} & \delta_{i_{4} i_{6}} & \delta_{i_{4} i_{7}} & \delta_{i_{4} i_{8}}
\end{array}\right|=0,
$$

which requires proper handling. In fact, the number of linearly independent fundamental Cartesian isotropic tensors of a given rank is given by Motzkin sum numbers, ${ }^{71}$ which for rank 8 is 91 rather than 105 , suggested by the double factorial derived for even rank above. Such considerations are not needed in the present approach, which in addition is well-suited for computer implementation.

\section{COMPUTATIONAL DETAILS}

Unless otherwise stated, the calculated results presented in this paper have been obtained by time-dependent density functional theory (TD-DFT) calculations, based on the Dirac-Coulomb Hamiltonian and within the restricted excitation window (REW) approach $^{72,73}$ using the PBE0 ${ }^{74,75}$ exchange-correlation functional and the dyall.ae $3 z$ basis sets. ${ }^{76,77}$ The small component basis sets were generated according to the condition of restricted kinetic balance, and the (SS|SS) integrals are replaced by an interatomic SS correction. ${ }^{78}$ A Gaussian model was employed for the nuclear charge distribution. ${ }^{79}$ A 86-point Lebedev grid $\left(L_{\max }=12\right)$ was used for 
TABLE I. Comparison of isotropically averaged oscillator strengths for $\mathrm{Cl} 1 s \rightarrow \mathrm{Ti} 3 d$ transitions of $\mathrm{TiCl}_{4}$ for the full semi-classical interaction operator and accumulated to various orders, as indicated by the superscripted number in parenthesis, within the multipolar gauge (Ir: length representation) and Coulomb gauge (vr: velocity representation), computed at the 4C-TD-PBE0 and Lévy-Leblond (LL) level of theory with different basis sets. Contributions from degenerate states have been summed. An 86-point $\left(L_{\mathrm{max}}=12\right)$ Lebedev grid was used to obtain the isotropically averaged full BED oscillator strengths. The gauge origin is placed on the Ti atom.

\begin{tabular}{|c|c|c|c|c|c|c|c|c|c|c|}
\hline Final state & $\Delta E(\mathrm{eV})$ & Gauge & $10^{3} f^{(\rightarrow 0)}$ & $10^{3} f^{(\rightarrow 2)}$ & $10^{3} f^{(\rightarrow 4)}$ & $10^{3} f^{(\rightarrow 6)}$ & $10^{3} f^{(\rightarrow 8)}$ & $10^{3} f^{(\rightarrow 10)}$ & $10^{3} f^{(\rightarrow 12)}$ & $10^{3} f_{\text {full }}$ \\
\hline \multicolumn{11}{|c|}{$6-31+\mathrm{G}^{*}-\mathrm{LL}$} \\
\hline \multirow{2}{*}{${ }^{1} T_{1}$} & \multirow{2}{*}{2763.004298} & lr & 0.000 & 16.616 & -6.599 & 7.867 & 2.748 & 3.913 & 3.730 & \multirow[t]{2}{*}{3.730} \\
\hline & & vr & 0.000 & 16.616 & -6.598 & 7.877 & 2.715 & 3.900 & 3.709 & \\
\hline \multirow{4}{*}{${ }^{1} E$} & $2763.004474^{a}$ & vr & 0.000 & 16.62 & $\ldots$ & $\cdots$ & $\cdots$ & $\cdots$ & $\cdots$ & \multirow{4}{*}{2.096} \\
\hline & \multirow{2}{*}{2763.004339} & $\operatorname{lr}$ & 0.000 & 6.762 & -1.188 & 3.360 & 1.814 & 2.164 & 2.112 & \\
\hline & & vr & 0.000 & 6.640 & -1.109 & 3.288 & 1.816 & 2.141 & 2.090 & \\
\hline & $2763.004515^{a}$ & vr & 0.000 & 6.64 & $\ldots$ & $\ldots$ & $\ldots$ & $\ldots$ & $\ldots$ & \\
\hline \multirow{5}{*}{${ }^{1} T_{2}$} & \multirow{2}{*}{2763.004306} & $\operatorname{lr}$ & 7.434 & -16.230 & 15.073 & -3.955 & 2.669 & 1.198 & 1.408 & \multirow[t]{3}{*}{1.396} \\
\hline & & vr & 7.246 & -16.033 & 14.988 & -3.943 & 2.690 & 1.180 & 1.422 & \\
\hline & $2763.004482^{a}$ & $\mathrm{vr}$ & 7.44 & -15.84 & $\cdots$ & $\cdots$ & $\cdots$ & $\cdots$ & $\cdots$ & \\
\hline & \multirow{2}{*}{ Sum } & lr & 7.434 & 7.147 & 7.286 & 7.273 & 7.231 & 7.275 & 7.249 & \multirow[t]{2}{*}{7.222} \\
\hline & & vr & 7.246 & 7.222 & 7.221 & 7.222 & 7.221 & 7.222 & 7.221 & \\
\hline \multicolumn{11}{|c|}{ dyall.ae3z-LL } \\
\hline \multirow{2}{*}{${ }^{1} T_{1}$} & \multirow{2}{*}{2762.623981} & $\operatorname{lr}$ & 0.000 & 17.964 & -7.142 & 8.505 & 2.959 & 4.230 & 4.026 & \multirow[t]{2}{*}{4.040} \\
\hline & & vr & 0.000 & 17.964 & -7.166 & 8.504 & 2.948 & 4.222 & 4.017 & \\
\hline \multirow{2}{*}{${ }^{1} E$} & \multirow{2}{*}{2762.623987} & $\operatorname{lr}$ & 0.000 & 7.192 & -1.179 & 3.553 & 1.976 & 2.322 & 2.269 & \multirow[t]{2}{*}{2.267} \\
\hline & & vr & 0.000 & 7.151 & -1.161 & 3.536 & 1.971 & 2.314 & 2.261 & \\
\hline \multirow{4}{*}{${ }^{1} T_{2}$} & \multirow{2}{*}{2762.623987} & $\operatorname{lr}$ & 7.880 & -17.335 & 16.149 & -4.230 & 2.893 & 1.276 & 1.533 & \multirow[t]{2}{*}{1.503} \\
\hline & & vr & 7.836 & -17.305 & 16.137 & -4.232 & 2.890 & 1.273 & 1.531 & \\
\hline & \multirow{2}{*}{ Sum } & lr & 7.880 & 7.821 & 7.828 & 7.829 & 7.828 & 7.828 & 7.828 & \multirow[t]{2}{*}{7.809} \\
\hline & & $\mathrm{vr}$ & 7.836 & 7.809 & 7.809 & 7.809 & 7.809 & 7.809 & 7.809 & \\
\hline \multicolumn{11}{|c|}{ dyall.ae $3 z-4 c$} \\
\hline \multirow{2}{*}{${ }^{1} T_{1}$} & \multirow{2}{*}{2773.351719} & $\operatorname{lr}$ & 0.000 & 17.976 & -7.344 & 8.560 & 2.879 & 4.191 & 3.979 & \multirow[t]{2}{*}{3.993} \\
\hline & & vr & 0.000 & 17.976 & -7.372 & 8.561 & 2.866 & 4.183 & 3.970 & \\
\hline${ }^{1} E$ & 2773.351723 & $\operatorname{lr}$ & 0.000 & 7.199 & -1.251 & 3.569 & 1.945 & 2.308 & 2.249 & 2.248 \\
\hline & $2 / 73.051 / 23$ & vr & 0.000 & 7.156 & -1.229 & 3.548 & 1.943 & 2.298 & 2.242 & \\
\hline${ }^{1} T_{2}$ & 2773.351725 & $\operatorname{lr}$ & 7.825 & -17.413 & 16.369 & -4.360 & 2.948 & 1.272 & 1.543 & 1.510 \\
\hline & 24.03 & vr & 7.781 & -17.380 & 16.353 & -4.358 & 2.942 & 1.271 & 1.540 & \\
\hline & Sum & lr & 7.825 & 7.763 & 7.775 & 7.769 & 7.772 & 7.772 & 7.771 & 7.752 \\
\hline & sum & vr & 7.781 & 7.752 & 7.752 & 7.752 & 7.752 & 7.752 & 7.752 & \\
\hline
\end{tabular}

${ }^{\mathrm{a}}$ Data in row taken from Ref. 20.

the isotropic averaging of the oscillator strengths based on the full light-matter interaction operator. The gauge origin was placed in the center-of-mass, and spatial symmetry was invoked in all cases except for the gauge-origin dependence calculations.

The geometry of $\mathrm{TiCl}_{4}$ was taken from Ref. 17 where it was obtained using the BP86 exchange-correlation functional ${ }^{80,81}$ and the TZP basis set. ${ }^{82}$ To enable a direct comparison to previous work, ${ }^{20}$ additional results on $\mathrm{TiCl}_{4}$ have been obtained using the non-relativistic Lévy-Leblond Hamiltonian ${ }^{83}$ employing a pointnucleus model and the $6-31+\mathrm{G}^{*}$ basis set, ${ }^{84-87}$ the latter as implemented in the Gaussian16 package. ${ }^{88}$

To study the apparent divergences of oscillator strengths for core excitations using truncated interaction, we carried out timedependent Hartree-Fock (TD-HF) calculations of $n s_{1 / 2} \rightarrow 7 p_{1 / 2}$ excitations of the radium atom. In these calculations, integral screening was turned off and the (SS|SS) integrals are included. 


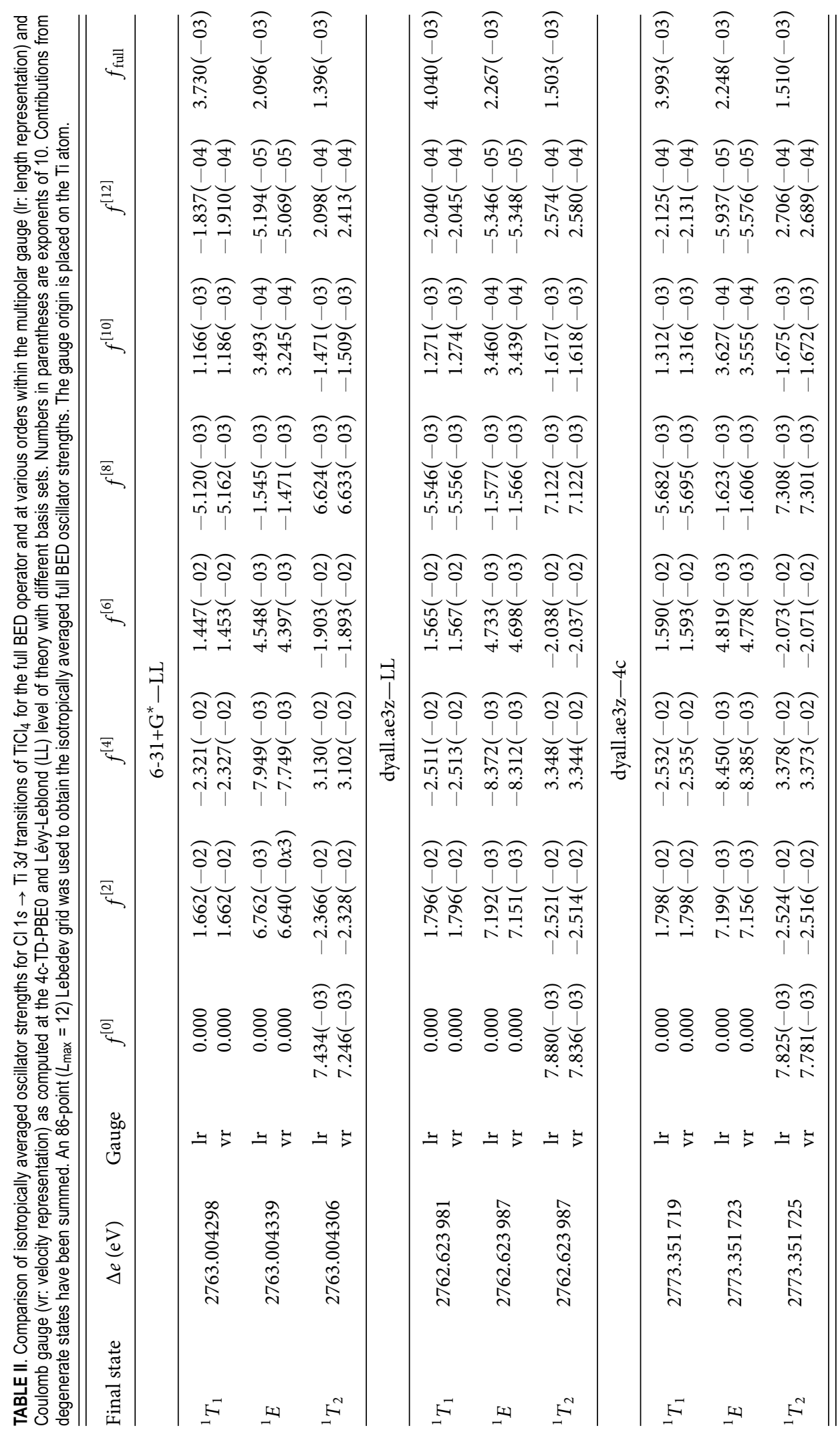


TABLE III. Gauge-origin dependency of the isotropically averaged oscillator strengths for the ${ }^{1} T_{2}$ set of $\mathrm{Cl} 1 s \rightarrow \mathrm{Ti} 3 d$ transitions of $\mathrm{TiCl}_{4}$ for the full semi-classical light-matter interaction operator and accumulated to various orders within the multipolar gauge (Ir: length representation) and Coulomb gauge (vr: velocity representation), computed at the 4c-TD-PBE0 level of theory and the dyall.ae3z basis set. Numbers in parentheses are exponents of 10 . At this level, the excitation energy is calculated as $2773.351145 \mathrm{eV}$. Contributions from the degenerate set have been summed. An 86-point $\left(L_{\max }=12\right)$ Lebedev grid was used to obtain the isotropically averaged full BED oscillator strengths. The gauge origin is shifted along the $x$-axis $\left(d_{x}\right)$ where $d_{x}=0.0 a_{0}$ corresponds to gauge-origin in the Ti atom.

\begin{tabular}{|c|c|c|c|c|c|c|c|c|c|}
\hline$d_{x}\left(a_{0}\right)$ & auge & $f^{(\rightarrow 0)}$ & $f^{(\rightarrow 2)}$ & $f^{(\rightarrow 4)}$ & $f^{(\rightarrow 6)}$ & $f^{(\rightarrow 8)}$ & $f^{(\rightarrow 10)}$ & $f^{(\rightarrow 12)}$ & $f_{\text {full }}$ \\
\hline 0 & $\begin{array}{l}\mathrm{lr} \\
\mathrm{vr}\end{array}$ & $\begin{array}{l}7.825(-03) \\
7.781(-03)\end{array}$ & $\begin{array}{l}-1.741(-02) \\
-1.738(-02)\end{array}$ & $\begin{array}{l}1.637(-02) \\
1.635(-02)\end{array}$ & $\begin{array}{l}-4.360(-03) \\
-4.358(-03)\end{array}$ & $\begin{array}{l}2.948(-03) \\
2.943(-03)\end{array}$ & $\begin{array}{l}1.272(-03) \\
1.271(-03)\end{array}$ & $\begin{array}{l}1.543(-03) \\
1.540(-03)\end{array}$ & 1.510 \\
\hline 10.0 & $\begin{array}{l}\text { lr } \\
\mathrm{vr}\end{array}$ & $\begin{array}{l}7.825(-03) \\
7.781(-03)\end{array}$ & $\begin{array}{l}-1.755(-02) \\
-1.738(-02)\end{array}$ & $\begin{array}{l}1.670(-02) \\
1.635(-02)\end{array}$ & $\begin{array}{l}-4.738(-03) \\
-4.358(-03)\end{array}$ & $\begin{array}{l}3.238(-03) \\
2.943(-03)\end{array}$ & $\begin{array}{l}1.097(-03) \\
1.271(-03)\end{array}$ & $\begin{array}{l}1.670(-03) \\
1.540(-03)\end{array}$ & $1.510(-03)$ \\
\hline 50.0 & $\begin{array}{l}\text { lr } \\
\mathrm{vr}\end{array}$ & $\begin{array}{l}7.825(-03) \\
7.781(-03)\end{array}$ & $\begin{array}{l}-2.045(-02) \\
-1.738(-02)\end{array}$ & $\begin{array}{l}1.422(-01) \\
1.635(-02)\end{array}$ & $\begin{array}{l}-2.951(+00) \\
-4.358(-03)\end{array}$ & $\begin{array}{l}4.429(+01) \\
2.943(-03)\end{array}$ & $\begin{array}{r}-5.055(+02) \\
1.271(-03)\end{array}$ & $\begin{array}{l}6.495(+03) \\
1.546(-03)\end{array}$ & $1.510(-03)$ \\
\hline 100.0 & $\begin{array}{l}\operatorname{lr} \\
\mathrm{vr}\end{array}$ & $\begin{array}{l}7.825(-03) \\
7.781(-03)\end{array}$ & $\begin{array}{l}-3.148(-02) \\
-1.738(-02)\end{array}$ & $\begin{array}{l}2.398(+00) \\
1.635(-02)\end{array}$ & $\begin{array}{l}-2.223(+02) \\
-4.358(-03)\end{array}$ & $\begin{array}{l}1.343(+04) \\
2.943(-03)\end{array}$ & $\begin{array}{r}-6.178(+05) \\
1.021(-03)\end{array}$ & $\begin{array}{l}3.198(+07) \\
4.785(-02)\end{array}$ & $1.510(-03)$ \\
\hline
\end{tabular}
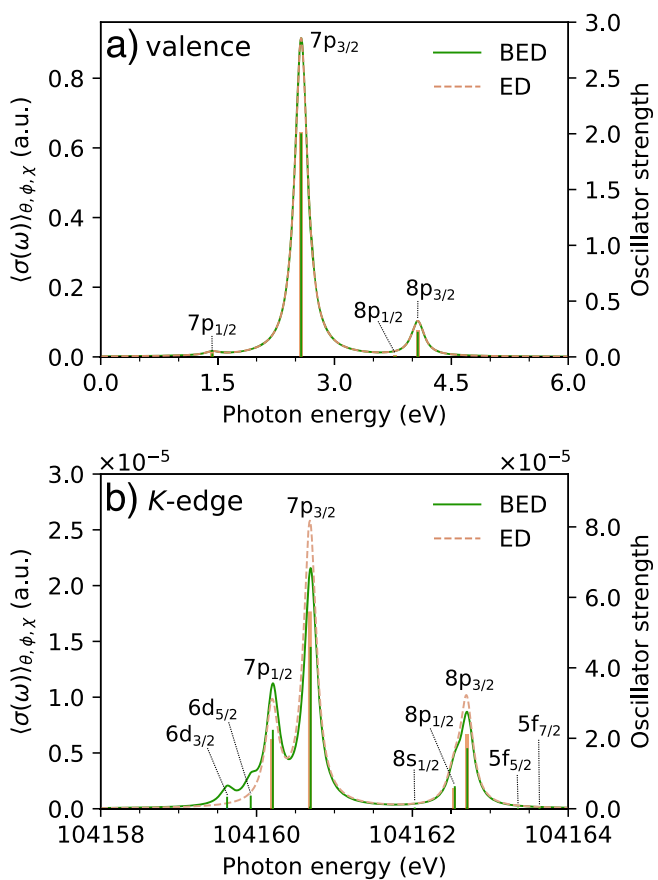

FIG. 1. Non-dipolar effects on electronic absorption of radium: (a) the valence and (b) K-edge spectra for Ra within and beyond the ED approximation (ED and BED, respectively) at the 4c-TD-PBE0/dyall.ae3z level of theory using the Coulomb gauge (velocity representation) for the former and the full interaction operator in Eq. (56) and an 86-point $\left(L_{\max }=12\right)$ Lebedev grid for the latter. The labels indicate the character of the receiving orbital. Note the differences in scales on the axes in the valence and $\mathrm{x}$-ray region. Oscillator strengths are summed over contributions from transitions within each degenerate (same $\Delta J$ components) and near-degenerate (different $\Delta J$ components) set, and the sticks have been convoluted with a Lorentzian lifetime broadening of $1000 \mathrm{~cm}^{-1}$. The experimental $1 \mathrm{~s}$ ionization energy is $103922 \pm 7.2 \mathrm{eV}$.
Unless otherwise stated, the data reported in this paper have been obtained with a development version of the DIRAC electronic structure code $^{32}$ (Tables I-III, Fig. 1: Revision 52c65be; Table IV, Fig. 2: Revision 5a7d81c).

\section{RESULTS AND DISCUSSION}

In this section, we demonstrate our implementation and study the behavior of the three presented schemes to go beyond the ED approximation. First, we consider the $\mathrm{Cl} \mathrm{K}$-edge in $\mathrm{TiCl}_{4}$, representing a case where there is no natural choice of gauge origin. It has previously been studied in the context of non-dipolar effects in linear x-ray absorption using low-order multipole expansions. In particular, it was used to demonstrate the appearance of negative oscillator strengths ${ }^{20}$ upon truncation of the light-matter interaction in the generalized velocity representation in a non-relativistic framework. ${ }^{17}$ Below, we will revisit this case. We further study numerically the gauge-origin dependence of the three schemes in the case of soft $x$-ray absorption. We then turn to their performance across the spectral range, including hard $\mathrm{x}$-rays, by considering atomic valence and core transitions in the radium atom. Given its high nuclear charge, radium shows strong relativistic effects both in the core and valence, and it is therefore a good example for comparing oscillator strengths within and beyond the ED approximation in a relativistic framework.

\section{A. $\mathrm{Cl} K$-edge absorption of $\mathrm{TiCl}_{4}$}

Ligand $K$-edge absorption spectroscopy supposedly provides direct information on the covalency of metal-ligand bonds due to the admixture of the ligand $p$-orbitals with the metal $d$ orbitals. ${ }^{89,90}$ The $\mathrm{Cl} K$-edge absorption of $\mathrm{TiCl}_{4}$ has been studied both experimentally and also theoretically within and beyond the ED approximation using truncated multipole-expanded expressions. 


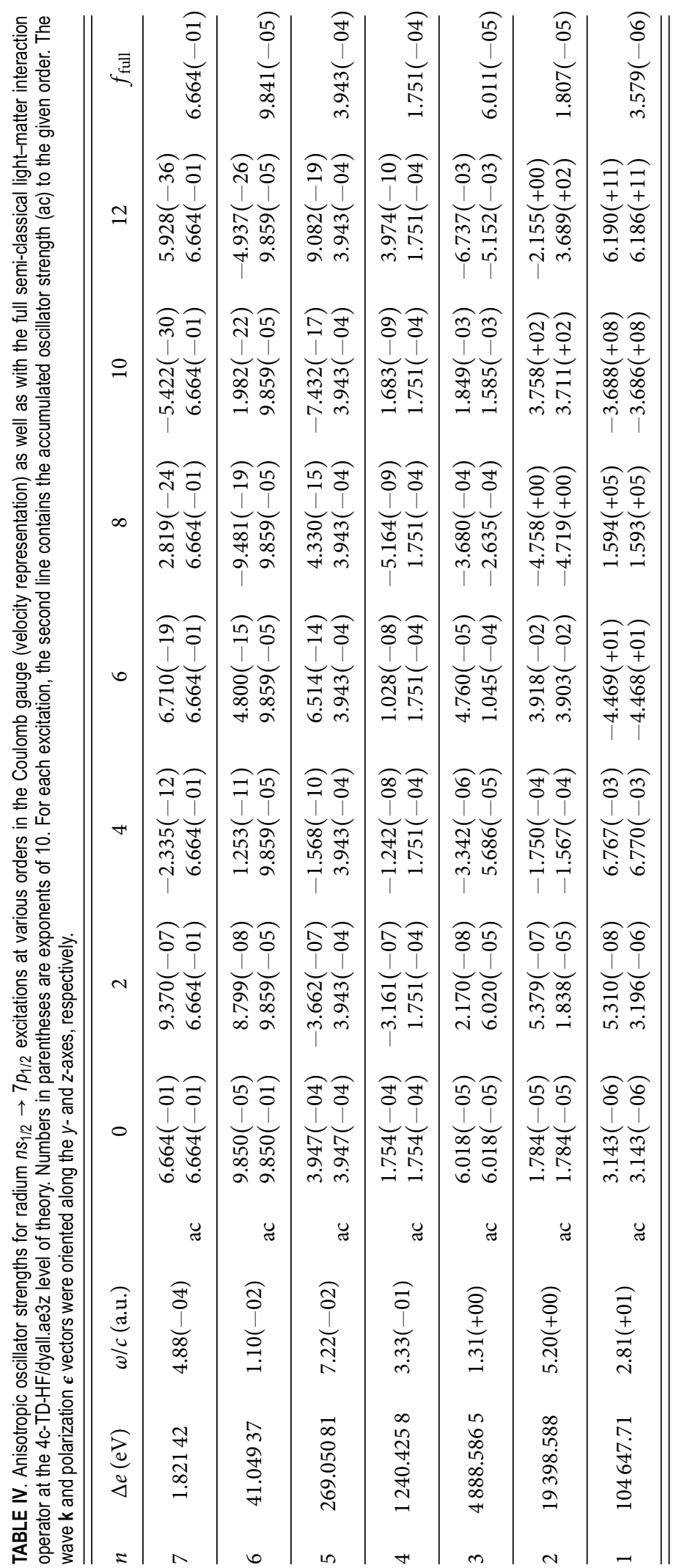




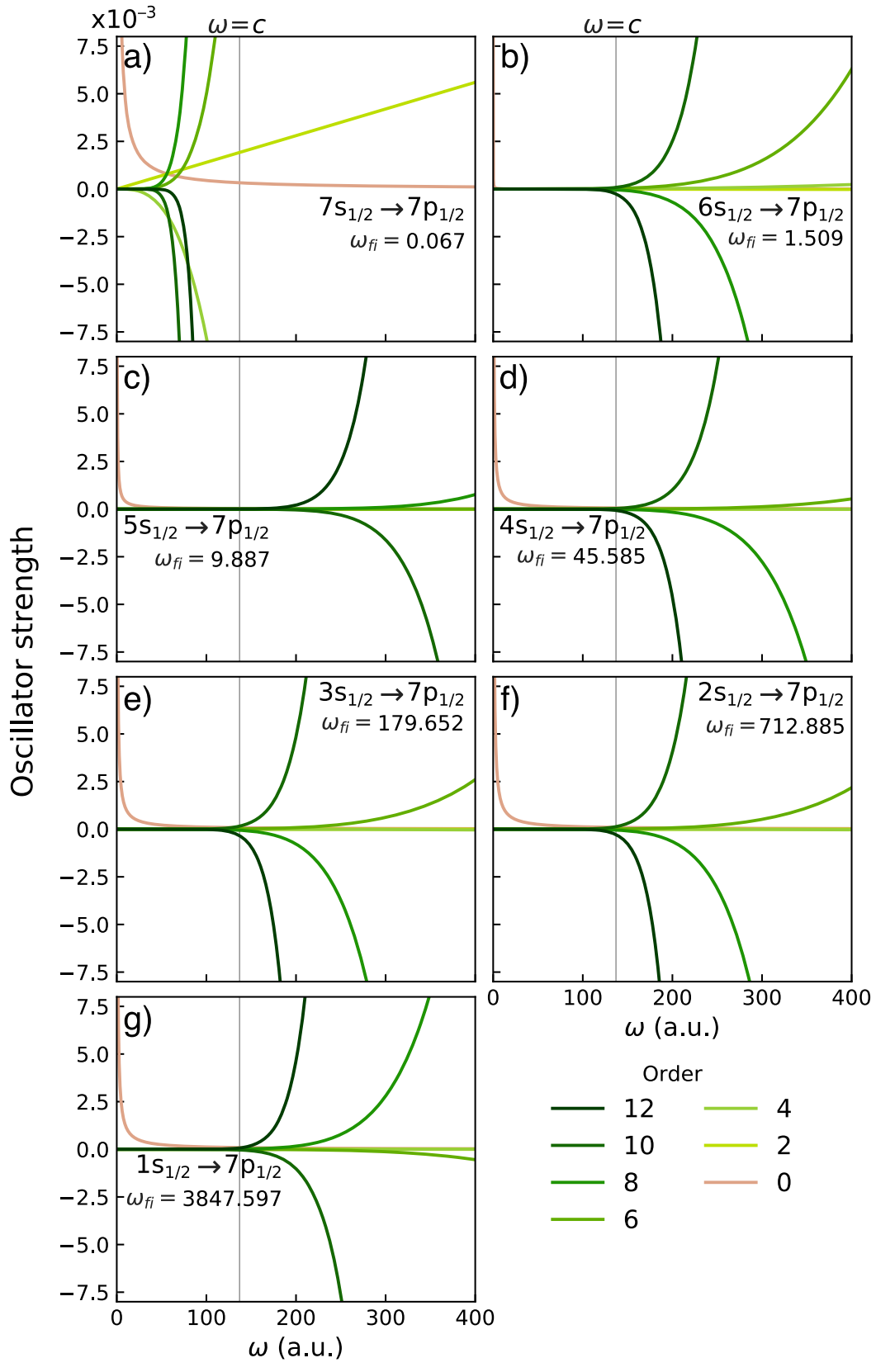

FIG. 2. Convergence behavior of the oscillator strengths for $n s_{1 / 2} \rightarrow 7 p_{1 / 2}$ transitions of radium at various orders (colored lines) in the wave vector within the Coulomb gauge (velocity representation): (a)-(g) correspond to $n=7,6, \ldots, 1$. Vertical dashed lines indicate $\omega=c$. Excitation energies $\left(\omega_{f i}\right)$ are in a.u.
Its experimental spectrum features a broad pre-edge peak that requires a two-peak fit (in toluene: at $2821.58 \mathrm{eV}$ and $2822.32 \mathrm{eV}$ with an approximate intensity ratio of 0.84$).{ }^{91}$ In $\mathrm{T}_{d}$ symmetry, the five $3 d$-orbitals of Ti belong to the $e$ and $t_{2}$ irreducible representations, and the pre-edge bands can be assigned to excitations from the $a_{1}$ and $t_{2} \mathrm{Cl} 1 \mathrm{~s}$-orbitals into the $e$ and $t_{2}$ sets of $3 d$-orbitals on $\mathrm{Ti}$, respectively. Here, we focus on the eight lowest-lying transitions $\left(a_{1}\right.$, $\left.t_{2} \rightarrow e\right)$, which give rise to three degenerate sets $\left(E, T_{1}\right.$, and $\left.T_{2}\right)$ of which the latter is ED allowed.

\section{Full vs truncated light-matter interaction}

Table I collects the isotropically averaged oscillator strengths for the pre-edge transitions computed in four-component relativistic and non-relativistic frameworks with the full light-matter interaction operator as well as accumulated to increasing orders (up to 12th order) in the wave vector within the Coulomb gauge (velocity representation) and multipolar gauge (length representation). First, we note that the trends are similar across the considered basis sets 
and Hamiltonians. In line with the results of Lestrange et al., ${ }^{20}$ we find negative oscillator strengths at the second order for the ${ }^{1} T_{2}$ excitations in both length and velocity representations. The same issue appears for the ${ }^{1} T_{1}$ and ${ }^{1} E$ sets, but at the fourth order. As discussed previously, ${ }^{20,21}$ this behavior is expected when the cross terms involving the lower-order moments to $f^{[n]}$ dominate the diagonal contributions. As evident from the underlying contributions given in Table II, the multipole expansions are alternating, and beyond fourth order, the correction is reduced at each order. Indeed, the expansions converge to the full expression at about 12th order irrespective of the employed basis set. For the dipole-allowed ${ }^{1} T_{2}$ set, the correction introduced by non-dipolar effects is significant, reducing the oscillator strength by a factor of $\sim 5$. As seen from the comparison of the ED and full (BED) oscillator strengths summed over the three sets of transitions, included in Table I, the implication of going beyond the ED approximation is a redistribution of intensity among transitions. In particular, the ED forbidden ${ }^{1} T_{1}$ and ${ }^{1} E$ transitions gain intensity beyond that of the $T_{2}$ set. We note, however, that this intensity redistribution has no consequence for the absorption band because of the near-degeneracy of the electronic transitions.

\section{Origin-dependence}

The above results were computed with the gauge-origin placed at the $\mathrm{Ti}$ atom. We now proceed to a numerical evaluation of their dependency on the gauge origin $(\mathbf{O}+\mathbf{a})$. As discussed above, the formulations based on the full semi-classical interaction operator and truncated interaction in the velocity representation are formally gauge invariant. In practical calculations, however, as discussed in Sec. II C 1, invariance in the latter case relies on the accurate cancellation of lower-order contributions multiplied with powers $(\mathbf{k} \cdot \mathbf{a})$, where $\mathbf{a}$ is the displacement. In contrast, as discussed in Sec. II C 2, in the multipolar gauge, formal gauge-origin invariance appears to only be achieved in the practically unreachable limit of the complete expansion of the fields.

Table III collects the total isotropic oscillator strength for the dipole-allowed ${ }^{1} T_{2}$ set for each of the three schemes for going beyond the ED approximation using different choices for the gauge origin. As expected, the results for the full light-matter interaction operator remain unchanged, providing a numerical verification of its gauge-origin invariance. The same is true for the oscillator strengths in the generalized velocity representation. However, numerical noise from the cancellation of many terms in powers of the displacement becomes apparent at large displacements. For a displacement of 100 $a_{0}$, instabilities start to appear at 10th order, and at 12th order, the oscillator strength exceeds the full result by one order of magnitude. This will be further discussed in Sec. IV B 2. The oscillator strengths in the multipolar gauge already at the second order differ significantly upon shifting the origin from the $\mathrm{Ti}$ atom.

\section{B. Radium}

\section{Full light-matter interaction}

In the valence region, the influence of non-dipolar effects is expected to be small except for ED forbidden transitions. Based on our previous study in a non-relativistic framework, we expect the effect on dipole-allowed core excitations to be modest $(\sim 10 \%)$ as a result of the compactness of the core hole.
Figure 1 shows the valence and $K$-edge spectra of Ra within and beyond the ED approximation, the latter computed with the full light-matter interaction operator. Expectedly, all ED forbidden transitions, except for excitations associated with change in total angular momentum quantum number $\Delta J=0$, gain intensity upon going beyond the ED approximation. In the valence region, however, they remain several orders of magnitude smaller than the ED counterparts such that ED and BED spectra are essentially identical. In the $\mathrm{x}$-ray region, the main contributions from the $1 s_{1 / 2} \rightarrow 6 d$ manifold correspond to $\Delta J=2$ transitions, while the ED allowed $\Delta J=1$ transitions dominate for the $1 s_{1 / 2} \rightarrow 7 / 8 p$ manifold. Note that the small energy differences between different $\Delta J$ components in a given set make them indiscernible in the spectrum, and we have therefore combined their oscillator strengths in Fig. 1. Upon inclusion of non-dipolar effects, intensity is primarily redistributed from the $1 s_{1 / 2} \rightarrow 7 / 8 p_{3 / 2}$ sets $(\mathrm{a} \sim 20 \%$ reduction compared to $\sim 13 \%$ for the $1 s_{1 / 2} \rightarrow 7 / 8 p_{1 / 2}$ excitations) to the $6 d$ transitions.

\section{Truncated light-matter interaction}

When carrying out equivalent calculations using the truncated light-matter interaction formulations, both in the velocity and the length representation, nonsensical results were obtained. Rather than reporting these numbers, we shall illustrate and analyze this behavior using a simpler computational setup. Table IV reports anisotropic oscillator strengths for radium $n s_{1 / 2} \rightarrow 7 p_{1 / 2}(n=1, \ldots$, 7) excitations at various orders in the generalized velocity representation as well as obtained using the full light-matter interaction. The orbital rotation operator [Eq. (A3)] is restricted to the $n s_{1 / 2}$ and the $7 p_{1 / 2}$ orbitals of the selected excitation, and we only report results for the $B_{1 u}$ irreducible representation of the $D_{2 h}$ point group. To avoid issues of numerical integration, we have performed TD-HF rather than TD-DFT calculations. Furthermore, to avoid possible numerical noise due to rotational averaging, we have chosen an oriented experiment, with the wave and polarization vectors oriented along the $y$ - and $z$-axes, respectively.

We see that for the $7 s_{1 / 2} \rightarrow 7 p_{1 / 2}$ excitation, the electric-dipole approximation holds since the zeroth-order oscillator strength $f^{[0]}$ reproduces the oscillator strength $f_{\text {full }}$, using the full interaction, to within the reported digits. For other excitations, the second-order oscillator strength $f^{[2]}$ has to be included in order to get reasonable agreement with the full interaction. For the $1 s_{1 / 2} \rightarrow 7 p_{1 / 2}$ transition, however, higher-order contributions to the oscillator strength blow up. A similar behavior, but to a lesser degree, is observed for the $2 s_{1 / 2}$ $\rightarrow 7 p_{1 / 2}$ transition, and we also note that the oscillator strength for the $3 s_{1 / 2} \rightarrow 7 p_{1 / 2}$ transition, accumulated to 12 th order, is negative. Very similar behavior is observed for the multipolar gauge (data not shown). In Table IV, we list for each excitation the corresponding norm $k=\omega / c$ of the wave vector. Interestingly, the apparent divergence in the expansion of the full light-matter interaction occurs when $k \approx 1 a_{0}^{-1}$. Indeed, if we do not set $\omega=\omega_{f i}$, where $\hbar \omega_{f i}$ is the excitation energy and instead treat $\omega$ as a variable, so as to artificially vary $k$ appearing in the interaction operator, we find that the oscillator strengths for all excitations blow up around $k=1 a_{0}^{-1}$ (Fig. 2). In passing, we note that the excitation energies for $\mathrm{Cl} 1 \mathrm{~s}$ $\rightarrow \mathrm{Ti} 3 d$ transitions of $\mathrm{TiCl}_{4}$ reported in Table I correspond to $k \approx 0.74 a_{0}^{-1}$. It seems reasonable that the convergence behavior of an expansion of oscillator strengths in orders of the norm of the 
wave vector should change when $k \approx 1 a_{0}^{-1}$. However, this conclusion requires some caution since $k$ is not a dimensionless quantity. The proper expansion parameter is rather the dimensionless quantity $k r$, and the above observations suggest that the effective radius $r \approx 1 a_{0}$. For the valence $7 s_{1 / 2} \rightarrow 7 p_{1 / 2}$ excitation, the effective radius $r$ is more diffuse, which explains why the apparent divergence sets in for $k<1 a_{0}^{-1}$, as shown in Fig. 2 .

The oscillator strengths of the given (even) order are calculated according to Eq. (30). We have also investigated to what extent transition moments over effective interaction operators $\hat{T}_{\text {full }}^{[n]}$ of order $n$ in the wave vector [Eq. (29)] sum up to transition moments over the full interaction operator and find apparent divergences for core excitations. Again, when treating $\omega$ as a variable and not setting it equal to $\omega_{f i}$, we find that these apparent divergences occur for all $n s_{1 / 2} \rightarrow 7 p_{1 / 2}$ excitations when $k>1 a_{0}^{-1}$. Going deeper in our analysis, we note that transition moments are obtained by contracting the property gradient of the selected operator with the solution vector for the selected excitation [Eq. (A14)]. Due to the restrictions on the orbital rotation operator in our particular case, the scalar product is reduced to the multiplication of two numbers. We find that an expansion of the property gradient of the full interaction in orders of the wave vector displays the same apparent divergence for core excitations as we observed for both oscillator strengths and transition moments. Again, by artificially varying $k$, we find that these apparent divergences occur when $k>1 a_{0}^{-1}$ for all excitations.

With our particular orientation of the experiment, the full and truncated effective interaction operators at order $n$ are given by

$$
\hat{T}_{\text {full }}(\omega)=\frac{e}{\omega} c \alpha_{z} e^{+i k y}, \quad \hat{T}_{\text {full }}^{[n]}(\omega)=\frac{e}{\omega} \frac{i^{n}}{n !} c \alpha_{z}(k y)^{n} .
$$

Elements of the property gradient [Eq. (A7)] of the truncated effective interaction operator are accordingly given by

$$
g_{T^{[n]} ; a i}=-\frac{e}{\omega}(i k)^{n}\left\{\left\langle\varphi_{a}^{L}\left|c \sigma_{z} \frac{y^{n}}{n !}\right| \varphi_{i}^{S}\right\rangle+\left\langle\varphi_{a}^{S}\left|c \sigma_{z} \frac{y^{n}}{n !}\right| \varphi_{i}^{L}\right\rangle\right\},
$$

where superscripts $L$ and $S$ refer to the large and small components of molecular orbital $\varphi_{p}$, respectively. In practice, as implemented in the DIRAC package, the property gradient is compounded from products of an atomic-orbital (AO) integral with two expansion coefficients on the form

$$
c_{\mu a}^{*}\left\langle\chi_{\mu}\left|c \frac{y^{n}}{n !}\right| \chi_{v}\right\rangle c_{v i}
$$

with the factor outside the curly brackets in Eq. (73) multiplied on at the end. In the present case, the coefficients are real due to symmetry. ${ }^{43}$ Each component of the Dirac spinor is expanded in Cartesian Gaussian-type orbitals (CGTOs),

$$
G_{i j k}^{\alpha}(\boldsymbol{r})=N_{i j k}^{\alpha} x^{i} y^{j} z^{k} e^{-\alpha r^{2}}, \quad i+j+k=\ell .
$$

For $n=12$, we find that the largest contribution, in terms of magnitude, to the property gradient comes from a small component $p_{y}$ function with exponent $\alpha_{1}=1.56556662(-02) a_{0}^{-2}$ combined with a large component $p_{y}$ function with exponent $\alpha_{2}$ $=1.24964369(-02) a_{0}^{-2}$. These are the most diffuse $s$ and $p$ functions, respectively, of the large component dyall.ae $3 z$ basis set. The resulting AO-integral has a value $-1.19437467(+6)$ a.u. and is multiplied with a coefficient $c_{1}=-4.55940113(-8)$ from $1 s_{1 / 2}$ and a coefficient $c_{2}=-0.844080786$ from $7 p_{1 / 2}$. By calculating AO-integrals with high precision using Mathematica, we find that the above AO-integrals, provided by the HERMIT integral package, ${ }^{94}$ are very stable. On the other hand, the very small $c_{1}$ coefficient is at the limits of the precision one can expect from the diagonalization of the Fock matrix, in particular, given its ill-conditioning due to the presence of negativeenergy solutions. We have, however, investigated the sensitivity of our results with respect to the HF convergence (in terms of the gradient) and find that they are quite stable at tight thresholds.

The final step of our analysis is to study the convergence of the AO-integrals over the truncated interaction toward the corresponding integral over the full interaction operator. Restricting attention to our particular case in Eq. (72) and Gaussian $p_{y}$ functions, the case in which only even-order terms contribute, we have

$$
\left\langle G_{010}^{\alpha_{1}}\left|\hat{T}_{\text {full }}(\omega)\right| G_{010}^{\alpha_{2}}\right\rangle=\sum_{m=0}^{\infty}\left\langle G_{010}^{\alpha_{1}}\left|\hat{T}_{\text {full }}^{[2 m]}(\omega)\right| G_{010}^{\alpha_{2}}\right\rangle .
$$

After eliminating common factors on both sides, we find an equivalent expression,

$$
-\left(4 Q^{2}-2\right) e^{-Q^{2}}=\sum_{m=0}^{\infty}(-1)^{m} a_{m}, \quad a_{m}=Q^{2 m} \frac{(2 m+2)(2 m+1)}{(m+1) !},
$$

in terms of a dimensionless parameter $Q$,

$$
Q=\frac{k}{2 \sqrt{\alpha_{1}+\alpha_{2}}} \text {. }
$$

(further details are given in Appendix D). The right-hand side expression has the form of an alternating series, and using the Leibniz criterion, we first note that $\lim _{m \rightarrow \infty} a_{m}=0$. On the other hand, the coefficients $a_{m}$ decrease monotonically only beyond a critical value of the summation index,

$$
m_{c}=\frac{1}{4}\left[\left(2 Q^{2}-3\right)+\sqrt{4 Q^{4}+12 Q^{2}+1}\right] .
$$

For the $1 s_{1 / 2} \rightarrow 7 p_{1 / 2}$ excitation and the above choice of exponents, we find that $m_{c} \approx Q^{2}=6998.7$. For this value of $Q$, the left-hand side of Eq. (77) is essentially zero, whereas the right-hand side converges extremely slowly toward this value. In fact, using Mathematica, ${ }^{93}$ no convergence was observed even after summing 10000 terms. Considering instead the $2 s_{1 / 2} \rightarrow 7 p_{1 / 2}$ excitation for which $m_{c} \approx 240$, reasonable convergence is found after summing 282 terms.

In summary, we have found that for increasing excitation energies, the use of truncated light-matter interaction becomes increasingly problematic because of the slow convergence of such expansions. This is not a problem that can be alleviated by increasing the basis set, since we observe this slow convergence at the level of the individual underlying AO-integrals. In particular, for core excitations, we have observed extremely slow convergence for integrals involving Cartesian Gaussian-type orbitals with diffuse exponents. This can be understood since such diffuse functions will be less efficient than tight ones in damping the increasing Cartesian powers appearing in an expansion of the full light-matter interaction in orders of the norm of the wave vector [see Eqs. (42) and (29)]. 
This in turn suggests that the use of Slater-type orbitals, which have slower decay than CGTOs, will be even more problematic. This is indeed the case as we show in Appendix D.

\section{CONCLUSION}

We have presented the implementation of three schemes for describing light-matter interactions beyond the electric-dipole approximation in the context of linear absorption within the fourcomponent relativistic domain: (i) the full semi-classical fieldmatter interaction operator in which the electric and magnetic interactions are included to all orders in the wave vector, in addition to two formulations based on a truncated interaction using either (ii) the Coulomb gauge (generalized velocity representation) or (iii) the multipolar gauge (generalized length representation). In the latter gauge, potentials are given in terms of the values of the electric and magnetic field and their derivatives at some expansion point. We have generalized the derivation of the multipolar gauge to arbitrary expansion points and shown that potentials associated with different expansion points are related by a gauge transformation but also that this is only guaranteed to the extent that the expansion is not truncated. We have further presented schemes for rotational averaging of the oscillator strength for each of the three cases. In particular, the simple form of the light-matter interaction operator in the relativistic formulation allowed for arbitrary-order implementations of the two truncated schemes with and without rotational averaging. We believe that this is a unique feature of our code.

Next, we have exploited the generality of our formulations and implementation to study, both analytically and numerically, the behavior of the two truncated schemes relative to the full lightmatter interaction with particular focus on the $\mathrm{x}$-ray spectral region. This analysis has highlighted the following important points:

- Oscillator strengths using truncated interaction in the Coulomb gauge (generalized velocity representation) are gauge-origin invariant at each order in the wave vector. This was originally shown in Ref. 17 but follows straightforwardly from our alternative derivation starting from a Taylor expansion of the full expression for the oscillator strength rather than that of the transition moments. A practical realization of this gauge-origin independence, however, relies on an accurate cancellation of terms multiplied by powers of the origin displacement. Thus, while origin invariance is numerically achievable at low frequencies and small displacements, it becomes increasingly difficult and even unreachable at higher frequencies and displacements.

- Formal gauge-origin invariance of oscillator strengths in the multipolar gauge hinges on commutator expressions that do not necessarily hold in a finite basis. This explains the notorious lack of order-by-order gauge-origin independence in practical calculations beyond the electric-dipole approximation based on any truncated multipolar gauge formulation. ${ }^{20,21}$ However, we would like to stress that these commutator relations, involving the Hamiltonian, correspond to a gauge transformation from the length to the velocity representation. In other words, gauge-origin independence in the multipolar gauge is shown by transforming to another gauge for which origin-independence holds. We have not been able to show gauge-origin invariance while staying within the multipolar gauge. An interesting feature of the multipolar gauge is that gauge freedom resides within the choice of expansion point. We show that a change of expansion point, that is, gauge origin, corresponds to a gauge transformation, but only if the expansion of the fields is not truncated.

- The appearance of negative oscillator strengths through the second order in the wave vector previously reported at the $\mathrm{Cl} \mathrm{K}$-edge for $\mathrm{TiCl}_{4}$ in the velocity representation ${ }^{20}$ is indeed a consequence of a too early truncation of the expansion, as previously suggested. ${ }^{20,22}$ In this case, convergence to the full light-matter interaction result is achieved at 12th order in the wave vector irrespective of the basis set used.

- While the oscillator strengths formulated using truncated interaction in the Coulomb gauge (velocity representation) are formally convergent across all frequencies, the series converges extremely slowly at high frequencies, an observation valid also for the multipolar gauge. We report a detailed investigation of a test case where we have studied convergence of the expansion in terms of the wave vector all the way from oscillator strengths to the underlying AO-integrals. For the latter quantities, the expansion in the dimensionless quantity $k r$ is replaced by an expansion in terms of the dimensionless quantity $Q=k / 2 \sqrt{\alpha_{1}+\alpha_{2}}$, where $\alpha_{1}$ and $\alpha_{2}$ are the Gaussian exponents. We find that the convergence of integrals over the truncated interaction toward integrals over the full interaction is extremely slow, requiring at least $Q^{2}$ terms. The convergence will depend on the decay of the basis functions. It will be particularly slow for diffuse exponents, as can be seen from the form of $Q$, and will be worse for Slater-type orbitals than for the Gaussiantype orbitals used in the present work. The onset of this complication is approximately defined by $\omega=c(\sim 3728 \mathrm{eV})$, although it also depends on the size of the given transition moments. Numerical instabilities using the Coulomb gauge in the generalized velocity representation can thus be expected already in the higher-energy end of the soft $\mathrm{x}$-ray region even though the onset may be delayed by the orderof-magnitude smaller transition moments associated with core excitations. Caution is therefore necessary while using this formulation in simulations of $\mathrm{x}$-ray absorption beyond the electric-dipole approximation because of its practical inapplicability beyond a certain frequency region.

The general numerical stability of the full light-matter interaction formulation to gauge-origin transformations and across frequencies as well as its ease of implementation in the context of linear absorption, demonstrated in this work and previously, $8,24,25$ makes this approach the method of choice for simulating linear absorption beyond the electric-dipole approximation. A possible complication of this approach, though, is that the underlying AO-integrals become dependent on the wave vector, hence excitation energies, and must generally be calculated on the fly.

\section{ACKNOWLEDGMENTS}

N.H.L. acknowledges financial support from the Carlsberg Foundation (Grant No. CF16-0290) and the Villum Foundation 
(Grant No. VKR023371). T.S. thanks Anthony Scemama (Toulouse) for the introduction to recursive loops. Computing time from CALMIP (Calcul en Midi-Pyrenées) and SNIC (Swedish National Infrastructure for Computing) at the National Supercomputer Centre (NSC) is gratefully acknowledged.

\section{DATA AVAILABILITY}

The data that support the findings of this study are available from the corresponding author upon reasonable request.

\section{APPENDIX A: SIMULATION OF ELECTRONIC SPECTRA FROM TIME-DEPENDENT RESPONSE THEORY}

In this appendix, we provide a brief overview of the simulation of electronic spectra using time-dependent Hartree-Fock (HF) theory as implemented in the DIRAC package ${ }^{32}$ under the restriction of a closed-shell reference. The formalism carries over with modest modifications to time-dependent Kohn-Sham (KS) theory. A fuller account is given in Ref. 95 and references therein.

We start from a Hamiltonian on the form

$$
\begin{gathered}
\hat{H}=\hat{H}_{0}+\hat{V}(t), \quad \hat{V}(t)=\sum_{k=-N}^{+N} \hat{V}\left(\omega_{k}\right) e^{-i \omega_{k} t}, \\
\hat{V}\left(\omega_{k}\right)=\sum_{X} \varepsilon_{X}\left(\omega_{k}\right) \hat{h}_{X}
\end{gathered}
$$

where $\varepsilon_{X}\left(\omega_{k}\right)$ represent the perturbation strengths. All frequencies $\omega_{k}$ are assumed to be integer multiples of a fundamental frequency $\omega_{T}=2 \pi / T$ such that the Hamiltonian is periodic of period $T$, allowing us to use the quasienergy formalism. ${ }^{46,96,97}$ We employ a unitary exponential parametrization of the closed-shell HF (or KS) determinant,

$$
|\tilde{0}(t)\rangle=\exp [-\hat{\kappa}(t)]|0\rangle,
$$

in terms of an anti-Hermitian, time-dependent orbital rotation operator

$$
\begin{aligned}
\hat{\kappa}(t) & =\sum_{a i}\left\{\kappa_{a i}(t) a_{a}^{\dagger} a_{i}-\kappa_{a i}^{*}(t) a_{i}^{\dagger} a_{a}\right\}, \\
\kappa_{p q}(t) & =\sum_{k=-N}^{+N} \kappa_{p q}\left(\omega_{k}\right) e^{-i \omega_{k} t} .
\end{aligned}
$$

Here and in the following, indices $(i, j \ldots),(a, b, \ldots)$, and $(p, q, \ldots)$ refer to occupied, virtual and general orbitals, respectively. The linear response of the system with respect to some perturbation $\hat{h}_{B}$ is found from the first-order response equation,

$$
\left(E_{0}^{[2]}-\hbar \omega_{b} S^{[2]}\right) \boldsymbol{X}_{B}\left(\omega_{b}\right)=-\boldsymbol{E}_{B}^{[1]},
$$

where

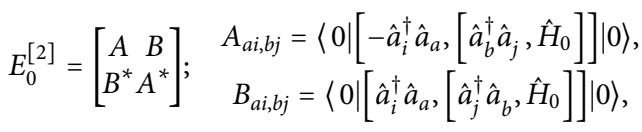

is the electronic Hessian,

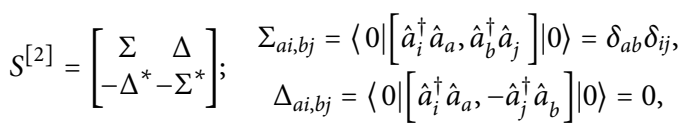

is the generalized metric, and

$$
\boldsymbol{E}_{B}^{[1]}=\left[\begin{array}{c}
\boldsymbol{g}_{B} \\
\Theta_{h B} \boldsymbol{g}_{B}^{*}
\end{array}\right] ; \quad g_{B ; a i}=-h_{B ; a i},
$$

is the property gradient. An important generalization above is that, in addition to Hermitian operators $\hat{h}_{B}\left(\Theta_{h B}=+1\right)$ imposed by the tenets of quantum mechanics, we also allow anti-Hermitian ones $\left(\Theta_{h B}=-1\right)$. It may seem awkward to speak about hermiticity of a vector, but the elements of the vector are, as seen from Eq. (A7), two-index quantities selected from a matrix and accordingly inherit the symmetries of that matrix.

The solution vector collects first-order frequency-dependent amplitudes,

$$
\begin{array}{cc}
\boldsymbol{X}_{B}\left(\omega_{b}\right)=\left[\begin{array}{c}
\boldsymbol{Z} \\
\left.\boldsymbol{Y}^{*}\right],
\end{array}\right. & Z_{a i}=\left[\frac{\partial \kappa_{a i}\left(\omega_{b}\right)}{\partial \varepsilon_{B}\left(\omega_{b}\right)}\right]_{\varepsilon=\mathbf{0}}=\kappa_{a i}^{B}\left(\omega_{b}\right), \\
& Y_{a i}=\left[\frac{\partial \kappa_{a i}\left(-\omega_{b}\right)}{\partial \varepsilon_{B}\left(\omega_{b}\right)}\right]_{\varepsilon=0}=\kappa_{a i}^{B}\left(-\omega_{b}\right),
\end{array}
$$

and linear response functions are obtained by contracting solution vectors with property gradients, that is,

$$
\langle\langle\hat{A} ; \hat{B}\rangle\rangle_{\omega_{b}}=\boldsymbol{E}_{A}^{[1] \dagger} \boldsymbol{X}_{B} .
$$

Excitation energies and corresponding transition moments, on the other hand, are found from the closely related general eigenvalue problem,

$$
\left(E_{0}^{[2]}-\hbar \lambda_{m} S^{[2]}\right) \boldsymbol{X}_{m}=0 .
$$

From the structure of the electronic Hessian $E_{0}^{[2]}$ [Eq. (A5)] and the general matrix $S^{[2]}[$ Eq. (A6)], it can be shown that solution vectors of both the first-order response equation [Eq. (A4)] and the eigenvalue equation [Eq. (A10)] come in pairs,

$$
\begin{aligned}
& \lambda_{+; m}=+\left|\omega_{m}\right|, \boldsymbol{X}_{+; m}=\left[\begin{array}{c}
\boldsymbol{Z}_{m} \\
\boldsymbol{Y}_{m}^{*}
\end{array}\right], \\
& \lambda_{-; m}=-\left|\omega_{m}\right|, \boldsymbol{X}_{-; m}=\left[\begin{array}{c}
\boldsymbol{Y}_{m} \\
\boldsymbol{Z}_{m}^{*}
\end{array}\right] .
\end{aligned}
$$

For Hermitian operators $\hat{h}_{A}$, transition moments are obtained by the contractions

$$
\begin{aligned}
& \left\langle 0\left|\hat{h}_{A}\right| n\right\rangle=\boldsymbol{E}_{A}^{[1] \dagger} \boldsymbol{X}_{+; n}=\boldsymbol{X}_{-; n}^{\dagger} \boldsymbol{E}_{A}^{[1]}, \\
& \left\langle n\left|\hat{h}_{A}\right| 0\right\rangle=\boldsymbol{X}_{+; n}^{\dagger} \boldsymbol{E}_{A}^{[1]}=\boldsymbol{E}_{A}^{[1] \dagger} \boldsymbol{X}_{-; n},
\end{aligned}
$$

A particular feature of the DIRAC package ${ }^{32}$ is that a symmetry scheme, based on quaternion algebra, is applied at the self-consistent field level and provides automatically the maximum point group and time-reversal symmetry reduction of the computational effort. ${ }^{43}$ However, the symmetry scheme is restricted to time-symmetric operators only since their matrix representations in a finite basis can be block diagonalized by a quaternion unitary transformation. ${ }^{98}$ In order to accommodate time-antisymmetric, Hermitian operators, they are made time-symmetric, anti-Hermitian by multiplication with imaginary $i,{ }^{99}$ that is,

$$
\hat{h}_{A} \rightarrow \hat{h}_{A^{\prime}}=i \hat{h}_{A} \quad \Rightarrow \quad \boldsymbol{E}_{A}^{[1]} \rightarrow \boldsymbol{E}_{A^{\prime}}^{[1]}=i \boldsymbol{E}_{A}^{[1]} .
$$


For consistency, we therefore have to generalize the above relations [Eq. (A12)] to

$$
\begin{aligned}
& \left\langle 0\left|\hat{h}_{A}\right| n\right\rangle=\Theta_{h A} \boldsymbol{E}_{A}^{[1] \dagger} \boldsymbol{X}_{+; n}=\boldsymbol{X}_{-; n}^{\dagger} \boldsymbol{E}_{A}^{[1]}, \\
& \left\langle n\left|\hat{h}_{A}\right| 0\right\rangle=\boldsymbol{X}_{+; n}^{\dagger} \boldsymbol{E}_{A}^{[1]}=\Theta_{h A} \boldsymbol{E}_{A}^{[1] \dagger} \boldsymbol{X}_{-; n} .
\end{aligned}
$$

An important observation is that whereas the matrix of timedependent amplitudes $\kappa_{p q}(t)$ is anti-Hermitian, the matrix of frequency-dependent amplitudes $\kappa_{p q}\left(\omega_{k}\right)$, from which solution vectors are built [cf. Eq. (A8)], is general, that is,

$$
\kappa_{p q}(t)=-\kappa_{q p}^{*}(t) \quad \Rightarrow \quad \kappa_{q p}^{*}\left(-\omega_{k}\right)=-\kappa_{p q}\left(\omega_{k}\right) .
$$

A key to computational efficiency is to consider a decomposition of solution vectors in terms of components of well-defined hermiticity and time reversal symmetry. ${ }^{46,99}$ Using a pair of solution vectors $\boldsymbol{X}_{+}$ and $\boldsymbol{X}_{-}$, we may form Hermitian and anti-Hermitian combinations,

$$
\begin{aligned}
& \boldsymbol{X}_{h}=\frac{1}{2}\left(\boldsymbol{X}_{+}+\boldsymbol{X}_{-}\right)=\left[\begin{array}{c}
\boldsymbol{Z}+\boldsymbol{Y} \\
\boldsymbol{Y}^{*}+\boldsymbol{Z}^{*}
\end{array}\right]=\left[\begin{array}{c}
\boldsymbol{h} \\
\boldsymbol{h}^{*}
\end{array}\right], \\
& \boldsymbol{X}_{a}=\frac{1}{2}\left(\boldsymbol{X}_{+}-\boldsymbol{X}_{-}\right)=\left[\begin{array}{c}
\boldsymbol{Z}-\boldsymbol{Y} \\
\boldsymbol{Y}^{*}-\boldsymbol{Z}^{*}
\end{array}\right]=\left[\begin{array}{c}
\boldsymbol{a} \\
\boldsymbol{a}^{*}
\end{array}\right] .
\end{aligned}
$$

The inverse relations therefore provide a separation of solution vectors into Hermitian and anti-Hermitian contributions,

$$
\boldsymbol{X}_{+}=\boldsymbol{X}_{h}+\boldsymbol{X}_{a}, \quad \boldsymbol{X}_{-}=\boldsymbol{X}_{h}-\boldsymbol{X}_{a} .
$$

Further decomposition of each contribution into time-symmetric and time-antisymmetric parts gives vectors that are well-defined with respect to both hermiticity and time reversal symmetry,

$$
\begin{gathered}
\boldsymbol{U}^{\dagger}\left(\Theta_{h}, \Theta_{t}\right)=\left[\begin{array}{lllll}
\boldsymbol{c}^{\dagger} & \boldsymbol{d}^{\dagger} \Theta_{t} \boldsymbol{c}^{T} \quad \Theta_{t} \boldsymbol{d}^{T} \Theta_{h} \boldsymbol{c}^{T} \Theta_{h} \boldsymbol{d}^{T} \Theta_{h} \Theta_{t} \boldsymbol{c}^{\dagger} \Theta_{h} \Theta_{t} \boldsymbol{d}^{\dagger}
\end{array}\right], \\
\left\{\begin{array}{l}
c_{a i}=x_{a i}, \\
d_{a i}=x_{a i},
\end{array}\right.
\end{gathered}
$$

where the index overbar refers to Kramers' partner in a Kramersrestricted orbital set. The scalar product of such vectors is given by ${ }^{46}$

$$
\begin{aligned}
\boldsymbol{U}_{1}^{\dagger}\left(\Theta_{h 1}, \Theta_{t 1}\right) \boldsymbol{U}_{2}\left(\Theta_{h 2}, \Theta_{t 2}\right) & =\left(1+\Theta_{h 1} \Theta_{h 2} \Theta_{t 1} \Theta_{t 2}\right)\left[z+\Theta_{h 1} \Theta_{h 2} z^{*}\right] ; \\
z & =\left(\boldsymbol{c}_{1}^{\dagger} \boldsymbol{c}_{2}+\boldsymbol{d}_{1}^{\dagger} \boldsymbol{d}_{2}\right),
\end{aligned}
$$

and one may therefore distinguish three cases

$$
\boldsymbol{U}_{1}^{\dagger}\left(\Theta_{h 1}, \Theta_{t 1}\right) \boldsymbol{U}_{2}\left(\Theta_{h 2}, \Theta_{t 2}\right)= \begin{cases}0, & \Theta_{h 1} \Theta_{h 2}=-\Theta_{t 1} \Theta_{t 2} \\ 4 \operatorname{Re}[z], & \Theta_{h 1} \Theta_{h 2}=\Theta_{t 1} \Theta_{t 2}=+1 \\ 4 i \operatorname{Im}[z], & \Theta_{h 1} \Theta_{h 2}=\Theta_{t 1} \Theta_{t 2}=-1 .\end{cases}
$$

One may show that hermiticity is conserved when multiplying a vector [Eq. (A19)] by the electronic Hessian, whereas it is reversed by the generalized metric. On the other hand, both the electronic Hessian and the generalized metric conserve time reversal symmetry. The implication is that the time-symmetric and time-antisymmetric components of a solution vector do not mix upon solving the generalized eigenvalue problem [Eq. (A10)] or the first-order response equation [Eq. (A4)] and one can dispense with one of them. From a physical point of view, this can be understood from the observation that excited states can be reached through both time-symmetric and time-antisymmetric operators. From a more practical point of view, this leads to computational savings corresponding to those obtained by re-expressing the generalized eigenvalue problem [Eq. (A10)] as a Hermitian one of half the dimension and involving the square of transition energies. Such a transformation can be done exactly in non-relativistic theory, ${ }^{100-102}$ but only through approximations in the relativistic domain. ${ }^{103,104}$ In the present scheme, we obtain the same computational savings without resorting to any transformations or approximations. In order to employ the quaternion symmetry scheme, we choose to work with the time-symmetric vectors. It follows from Eq. (A21) that their scalar products are either zero or real. In practice, a property gradient is therefore always contracted with the component of the solution vector having the same hermiticity so that all transition moments are real.

\section{APPENDIX B: MULTIPOLAR GAUGE}

In this appendix, we present a compact derivation of the multipolar gauge, following to a large extent Bloch ${ }^{47}$ and avoiding indices. We shall write the Taylor expansion of the scalar and vector potential about a reference point $\mathbf{a}$ as

$$
\begin{aligned}
& \tilde{\phi}(\mathbf{r}, t)=\sum_{n=0}^{\infty} \frac{1}{n !}\left[\left(\boldsymbol{\delta}_{\mathrm{a}} \cdot \nabla^{\prime}\right)^{n} \tilde{\phi}\left(\mathbf{r}^{\prime}, t\right)\right]_{\mathbf{r}^{\prime}=\mathbf{a}}, \quad \boldsymbol{\delta}_{\mathrm{a}}=\mathbf{r}-\mathbf{a} . \\
& \tilde{\mathbf{A}}(\mathbf{r}, t)=\sum_{n=0}^{\infty} \frac{1}{n !}\left[\left(\boldsymbol{\delta}_{\mathrm{a}} \cdot \nabla^{\prime}\right)^{n} \tilde{\mathbf{A}}\left(\mathbf{r}^{\prime}, t\right)\right]_{\mathbf{r}^{\prime}=\mathbf{a}},
\end{aligned}
$$

We then use the relation $\mathbf{E}=-\nabla \phi-\partial_{t} \mathbf{A}$ to rewrite the scalar potential as

$$
\begin{aligned}
\tilde{\phi}(\mathbf{r}, t)= & \tilde{\phi}(\mathbf{a}, t)-\sum_{n=1}^{\infty} \frac{1}{n !}\left[\left(\boldsymbol{\delta}_{\mathrm{a}} \cdot \nabla^{\prime}\right)^{n-1}\left(\boldsymbol{\delta}_{\mathbf{a}} \cdot \mathbf{E}\left(\mathbf{r}^{\prime}, t\right)\right)\right]_{\mathbf{r}^{\prime}=\mathbf{a}} \\
& -\partial_{t} \sum_{n=1}^{\infty} \frac{1}{n !}\left[\left(\boldsymbol{\delta}_{\mathrm{a}} \cdot \nabla^{\prime}\right)^{n-1}\left(\boldsymbol{\delta}_{\mathrm{a}} \cdot \tilde{\mathbf{A}}\left(\mathbf{r}^{\prime}, t\right)\right)\right]_{\mathbf{r}^{\prime}=\mathbf{a}} .
\end{aligned}
$$

The scalar potential now has the form of a gauge transformation,

$$
\tilde{\phi}(\mathbf{r}, t)=\phi(\mathbf{r}, \mathbf{t})-\partial_{t} \chi(\mathbf{r}, \mathbf{t}),
$$

where the gauge function $\chi$ is given by

$$
\chi(\mathbf{r}, t)=\sum_{n=1}^{\infty} \frac{1}{n !}\left[\left(\boldsymbol{\delta}_{\mathrm{a}} \cdot \nabla^{\prime}\right)^{n-1}\left(\boldsymbol{\delta}_{\mathrm{a}} \cdot \tilde{\mathbf{A}}\left(\mathbf{r}^{\prime}, t\right)\right)\right]_{\mathbf{r}^{\prime}=\mathbf{a}} .
$$

Using the partner relation

$$
\tilde{\mathbf{A}}(\mathbf{r}, t)=\mathbf{A}(\mathbf{r}, \mathbf{t})+\nabla \chi(\mathbf{r}, \mathbf{t})
$$

we first work out the gradient of the gauge function to be

$$
\begin{aligned}
\nabla \chi(\mathbf{r}, t)= & \sum_{n=0}^{\infty} \frac{1}{(n+1) !}\left[\left(\boldsymbol{\delta}_{\mathrm{a}} \cdot \nabla^{\prime}\right)^{n} \tilde{\mathbf{A}}\left(\mathbf{r}^{\prime}, t\right)\right]_{\mathbf{r}^{\prime}=\mathbf{a}} \\
& +\sum_{n=1}^{\infty} \frac{n}{(n+1) !}\left[\left(\boldsymbol{\delta}_{\mathbf{a}} \cdot \nabla^{\prime}\right)^{n-1} \nabla^{\prime}\left(\boldsymbol{\delta}_{\mathbf{a}} \cdot \tilde{\mathbf{A}}\left(\mathbf{r}^{\prime}, t\right)\right)\right]_{\mathbf{r}^{\prime}=\mathbf{a}}
\end{aligned}
$$


Further manipulation then gives

$$
\begin{aligned}
\mathbf{A}(\mathbf{r}, \mathbf{t})= & \sum_{n=1}^{\infty} \frac{n}{(n+1) !} \\
& \times\left[\left(\boldsymbol{\delta}_{\mathrm{a}} \cdot \nabla^{\prime}\right)^{n-1}\left\{\left(\boldsymbol{\delta}_{\mathrm{a}} \cdot \nabla^{\prime}\right) \tilde{\mathbf{A}}\left(\mathbf{r}^{\prime}, t\right)-\nabla^{\prime}\left(\boldsymbol{\delta}_{\mathrm{a}} \cdot \tilde{\mathbf{A}}\left(\mathbf{r}^{\prime}, t\right)\right)\right\}\right]_{\mathbf{r}^{\prime}=\mathbf{a}} .
\end{aligned}
$$

Finally, using the relation

$$
\boldsymbol{\delta} \times \mathbf{B}=\boldsymbol{\delta} \times(\nabla \times \tilde{\mathbf{A}})=\nabla(\boldsymbol{\delta} \cdot \tilde{\mathbf{A}})-(\boldsymbol{\delta} \cdot \nabla) \tilde{\mathbf{A}},
$$

we arrive at the final form of the potentials

$$
\begin{aligned}
& \phi_{\mathrm{a}}(\mathbf{r}, t)=\tilde{\phi}(\mathbf{a}, t)-\sum_{n=0}^{\infty} \frac{1}{(n+1) !}\left[\left(\boldsymbol{\delta}_{\mathrm{a}} \cdot \nabla^{\prime}\right)^{n}\left(\boldsymbol{\delta}_{\mathrm{a}} \cdot \mathbf{E}\left(\mathbf{r}^{\prime}, t\right)\right)\right]_{\mathbf{r}^{\prime}=\mathbf{a}}, \\
& \mathbf{A}_{\mathrm{a}}(\mathbf{r}, \mathbf{t})=-\sum_{n=1}^{\infty} \frac{n}{(n+1) !}\left[\left(\boldsymbol{\delta}_{\mathrm{a}} \cdot \nabla^{\prime}\right)^{n-1}\left(\boldsymbol{\delta}_{\mathrm{a}} \times \mathbf{B}\left(\mathbf{r}^{\prime}, t\right)\right)\right]_{\mathbf{r}^{\prime}=\mathbf{a}} .
\end{aligned}
$$

An alternative derivation of the multipolar gauge, ${ }^{44,49,105,106}$ which we here generalize to an arbitrary expansion point, is obtained by integrating Eq. (B5) along a line from expansion point a to observer point $\boldsymbol{r}$ and setting the gauge condition

$$
\boldsymbol{\delta}_{\mathrm{a}} \cdot \int_{0}^{1} \mathbf{A}\left(\lambda \boldsymbol{\delta}_{\mathrm{a}}+\mathbf{a}, t\right) d \lambda=0 .
$$

The gauge function is then found to be

$$
\chi_{\mathrm{a}}(\mathbf{r}, \mathbf{t})=\int_{0}^{1} \boldsymbol{\delta}_{\mathrm{a}} \cdot \tilde{\mathbf{A}}\left(\lambda \boldsymbol{\delta}_{\mathrm{a}}+\mathbf{a}, t\right) d \lambda,
$$

and the resulting potentials read

$$
\begin{aligned}
& \phi_{\mathrm{a}}(\mathbf{r}, t)=\tilde{\phi}(\mathbf{a}, t)-\boldsymbol{\delta}_{\mathrm{a}} \cdot \int_{0}^{1} \mathbf{E}\left(\lambda \boldsymbol{\delta}_{\mathrm{a}}+\mathbf{a}, t\right) d \lambda, \\
& \mathbf{A}_{\mathrm{a}}(\mathbf{r}, \mathbf{t})=-\int_{0}^{1} \lambda\left[\boldsymbol{\delta}_{\mathrm{a}} \times \mathbf{B}\left(\lambda \boldsymbol{\delta}_{\mathrm{a}}+\mathbf{a}, t\right)\right] d \lambda,
\end{aligned}
$$

where we have used Eq. (B8). The equivalence of the expressions of the present paragraph with those of the preceding one is seen by expanding the functions of $\mathbf{r}^{\prime}=\lambda \boldsymbol{\delta}_{\mathbf{a}}+\mathbf{a}$ in the integrands about $\mathbf{r}^{\prime}=\mathbf{a}$.

In passing, we note that the divergence of the vector potential is given by

$$
\begin{aligned}
\nabla \cdot \mathbf{A}(\mathbf{r}, \mathbf{t}) & =\int_{0}^{1} \lambda \boldsymbol{\delta}_{\mathrm{a}} \cdot\left(\nabla \times \mathbf{B}\left(\lambda \boldsymbol{\delta}_{\mathrm{a}}+\mathbf{a}, t\right)\right) d \lambda \\
& =\int_{0}^{1} \lambda^{2} \boldsymbol{\delta}_{\mathrm{a}} \cdot\left(\mu_{0} \mathbf{j}\left(\lambda \boldsymbol{\delta}_{\mathrm{a}}+\mathbf{a}, t\right)+\frac{1}{c^{2}} \partial_{t} \mathbf{E}\left(\lambda \boldsymbol{\delta}_{\mathrm{a}}+\mathbf{a}, t\right)\right) d \lambda
\end{aligned}
$$

where $\mu_{0}$ is the magnetic constant and $\mathbf{j}$ is the current density. The Ampère-Maxwell law was used in the final step. This relation shows that the multipolar gauge is equivalent to the Coulomb gauge only in the absence of external currents and for static electric fields.

In the multipolar gauge, the potentials are given in terms of the fields and their derivatives at the selected expansion point, which seems to eliminate any gauge freedom. However, this is incorrect. The gauge freedom is retained in the free choice of the expansion point. Consider now the gauge transformation taking us from potentials $\left(\mathbf{A}_{\mathrm{a}}, \phi_{\mathrm{a}}\right)$, defined with respect to expansion point $\mathbf{a}$, to a new set of potentials $\left(\mathbf{A}_{b}, \phi_{b}\right)$, defined with respect to expansion point $\mathbf{b}$. Clearly, the gauge function $\chi_{\mathrm{a} \rightarrow \mathrm{b}}$ satisfies

$$
\begin{aligned}
& \partial_{t} \chi_{\mathrm{a} \rightarrow \mathrm{b}}(\mathbf{r}, \mathbf{t})=\phi_{\mathrm{a}}(\mathbf{r}, t)-\phi_{\mathrm{b}}(\mathbf{r}, \mathbf{t}), \\
& \nabla \chi_{\mathrm{a} \rightarrow \mathrm{b}}(\mathbf{r}, \mathbf{t})=\mathbf{A}_{\mathrm{b}}(\mathbf{r}, t)-\mathbf{A}_{\mathrm{a}}(\mathbf{r}, t) .
\end{aligned}
$$

Starting from Eq. (B15), we find that

$$
\begin{aligned}
\nabla \partial_{t} \chi_{\mathrm{a} \rightarrow \mathrm{b}}(\mathbf{r}, \mathbf{t})= & \sum_{n=1}^{\infty} \frac{n}{(n+1) !}\left\{\left[\left(\boldsymbol{\delta}_{\mathrm{b}} \cdot \nabla^{\prime}\right)^{n-1} \nabla^{\prime}\left(\boldsymbol{\delta}_{\mathrm{b}} \cdot \mathbf{E}\left(\mathbf{r}^{\prime}, t\right)\right)\right]_{\mathbf{r}^{\prime}=\mathbf{b}}\right. \\
& \left.-\left[\left(\boldsymbol{\delta}_{\mathrm{a}} \cdot \nabla^{\prime}\right)^{n-1} \nabla^{\prime}\left(\boldsymbol{\delta}_{\mathrm{a}} \cdot \mathbf{E}\left(\mathbf{r}^{\prime}, t\right)\right)\right]_{\mathbf{r}^{\prime}=\mathbf{a}}\right\} \\
& +\sum_{n=0}^{\infty} \frac{1}{(n+1) !}\left\{\left[\left(\boldsymbol{\delta}_{\mathrm{b}} \cdot \nabla^{\prime}\right)^{n} \mathbf{E}\left(\mathbf{r}^{\prime}, t\right)\right]_{\mathbf{r}^{\prime}=\mathbf{b}}\right. \\
& \left.-\left[\left(\boldsymbol{\delta}_{\mathrm{a}} \cdot \nabla^{\prime}\right)^{n} \mathbf{E}\left(\mathbf{r}^{\prime}, t\right)\right]_{\mathbf{r}^{\prime}=\mathbf{a}}\right\} .
\end{aligned}
$$

On the other hand, starting from Eq. (B16), we find that

$$
\begin{aligned}
\partial_{t} \nabla \chi_{\mathrm{a} \rightarrow \mathrm{b}}(\mathbf{r}, \mathbf{t})= & \sum_{n=1}^{\infty} \frac{n}{(n+1) !}\left\{\left[\left(\boldsymbol{\delta}_{\mathrm{b}} \cdot \nabla^{\prime}\right)^{n-1} \nabla^{\prime}\left(\boldsymbol{\delta}_{\mathrm{b}} \cdot \mathbf{E}\left(\mathbf{r}^{\prime}, t\right)\right)\right]_{\mathbf{r}^{\prime}=\mathbf{b}}\right. \\
& \left.-\left[\left(\boldsymbol{\delta}_{\mathrm{a}} \cdot \nabla^{\prime}\right)^{n-1} \nabla^{\prime}\left(\boldsymbol{\delta}_{\mathrm{a}} \cdot \mathbf{E}\left(\mathbf{r}^{\prime}, t\right)\right)\right]_{\mathbf{r}^{\prime}=\mathbf{a}}\right\} \\
& -\sum_{n=1}^{\infty} \frac{n}{(n+1) !}\left\{\left[\left(\boldsymbol{\delta}_{\mathrm{b}} \cdot \nabla^{\prime}\right)^{n} \mathbf{E}\left(\mathbf{r}^{\prime}, t\right)\right]_{\mathbf{r}^{\prime}=\mathbf{b}}\right. \\
& \left.-\left[\left(\boldsymbol{\delta}_{\mathrm{a}} \cdot \nabla^{\prime}\right)^{n} \mathbf{E}\left(\mathbf{r}^{\prime}, t\right)\right]_{\mathbf{r}^{\prime}=\mathbf{a}}\right\},
\end{aligned}
$$

where we have used Faraday's law

$$
\nabla \times \mathbf{E}+\partial_{t} \mathbf{B}=0 .
$$

Due to the commutation of space and time derivatives, the two expressions should be the same, provided that the potentials at the two expansion points are related by a gauge transformation. At first sight, this does not seem to be the case since the second sum of the above expressions differ. However, actually calculating the difference gives

$$
\begin{aligned}
\nabla \partial_{t} \chi_{\mathrm{a} \rightarrow \mathrm{b}}(\mathbf{r}, \mathbf{t})-\partial_{t} \nabla \chi_{\mathrm{a} \rightarrow \mathrm{b}}(\mathbf{r}, \mathbf{t})= & \sum_{n=0}^{\infty} \frac{1}{n !}\left\{\left[\left(\boldsymbol{\delta}_{\mathrm{b}} \cdot \nabla^{\prime}\right)^{n} \mathbf{E}\left(\mathbf{r}^{\prime}, t\right)\right]_{\mathbf{r}^{\prime}=\mathbf{b}}\right. \\
& \left.-\left[\left(\boldsymbol{\delta}_{\mathrm{a}} \cdot \nabla^{\prime}\right)^{n} \mathbf{E}\left(\mathbf{r}^{\prime}, t\right)\right]_{\mathbf{r}^{\prime}=\mathbf{a}}\right\}=0,
\end{aligned}
$$

which is zero since the final line is the difference of the Taylor expansions of $\mathbf{E}(\mathbf{r}, t)$ at the two different expansion points. However, a very important observation is that this cancellation, and hence gauge freedom, is only assured if the expansions are not truncated.

Before closing this brief overview of the multipolar gauge, we remark that in some sources, a distinction is made between minimal coupling and multipolar Hamiltonians. ${ }^{18,107-111}$ This terminology arises from the observation that gauge transformations in quantum mechanics (also beyond) may be induced by a local unitary transformation of the wave function ${ }^{52,112,113}$

$$
\psi(\mathbf{r}, t) \rightarrow \psi^{\prime}(\mathbf{r}, t)=U(\mathbf{r}, t) \psi(\mathbf{r}, t) ; \quad U(\mathbf{r}, t)=e^{-\frac{i}{\hbar} q \chi(\mathbf{r}, t)},
$$

where $q$ is the particle charge, with the corresponding timedependent wave equation

$$
\left(\hat{\mathcal{H}}(\mathbf{A}, \phi)-i \hbar \partial_{t}\right) \psi(\mathbf{r}, t)=0 \quad \rightarrow \quad\left(\hat{\mathcal{H}}^{\prime}\left(\mathbf{A}^{\prime}, \phi^{\prime}\right)-i \hbar \partial_{t}\right) \psi^{\prime}(\mathbf{r}, t)=0
$$


expressed in terms of a transformed Hamiltonian

$$
\hat{\mathcal{H}}^{\prime}=U \hat{\mathcal{H}} U^{-1}-i \hbar U \partial_{t}\left(U^{-1}\right)=U \hat{\mathcal{H}} U^{-1}+q \partial_{t} \chi
$$

with potentials

$$
\mathbf{A}^{\prime}=\mathbf{A}-\nabla \chi, \quad \phi^{\prime}=\phi+\partial_{t} \chi .
$$

Accordingly, the multipolar or Power-Zienau-Woolley Hamiltonian $^{114-118}$ is obtained from transforming the non-relativistic minimal coupling Hamiltonian by using the multipolar gauge function [Eq. (B4)]. However, this is possibly misleading terminology since minimal coupling is a general procedure for coupling particles to fields, ${ }^{33,46}$ and indeed, the multipolar Hamiltonian can equivalently be obtained by plugging in the multipolar gauge potentials [Eq. (B9)] into the free-particle Hamiltonian according to the principle of minimal electromagnetic coupling. ${ }^{109,119}$

A final observation is that the transformed Hamiltonian [Eq. (B22)] using the Baker-Campbell-Hausdorff (BCH) expansion can alternatively be expressed as a sequence of increasingly nested commutators involving the gauge function and the original Hamiltonian

$$
\hat{\mathcal{H}}^{\prime}=\hat{\mathcal{H}}+q \partial_{t} \chi-q \frac{i}{\hbar}[\chi, \hat{\mathcal{H}}]-\frac{q^{2}}{2 \hbar^{2}}[\chi,[\chi, \hat{\mathcal{H}}]]+\ldots
$$

An illuminating example is to start from the gauge function associated with multipolar gauge [Eq. (B4)]. If we introduce the potentials [Eq. (17)] associated with linearly polarized monochromatic light in the Coulomb gauge, the gauge function for expansion point $\mathbf{a}=\mathbf{0}$ can be expressed as

$$
\begin{aligned}
\chi(\mathbf{r}, t) & =\chi(\mathbf{r}, \omega) e^{-i \omega t}+\chi(\mathbf{r},-\omega) e^{+i \omega t} \\
\chi(\mathbf{r}, \omega) & =-\frac{E_{\omega}}{2 \omega} \sum_{n=0}^{\infty} \frac{1}{(n+1) !}(i \mathbf{k} \cdot \mathbf{r})^{n}(\mathbf{r} \cdot \boldsymbol{\epsilon}) e^{i \delta} .
\end{aligned}
$$

Using Eq. (48), we find

$$
\begin{aligned}
-\frac{i}{\hbar}[\chi(\mathbf{r}, \omega), \hat{\mathcal{H}}]= & -\frac{E_{\omega}}{2 \omega} \sum_{n=0}^{\infty} \frac{1}{(n+1) !}(c \boldsymbol{\alpha} \cdot \boldsymbol{\epsilon})(i \mathbf{k} \cdot \mathbf{r})^{n} e^{i \delta} \\
& -\frac{E_{\omega}}{2 \omega} \sum_{n=0}^{\infty} \frac{n}{(n+1) !}(\mathbf{r} \cdot \boldsymbol{\epsilon})(i \mathbf{k} \cdot c \boldsymbol{\alpha})(i \mathbf{k} \cdot \mathbf{r})^{n-1} e^{i \delta}
\end{aligned}
$$

whereas $[\chi,[\chi, \hat{\mathcal{H}}]]$ and all higher-order commutators in the $\mathrm{BCH}$ expansion vanish, as can be seen from Eqs. (B25) and (B26).

Starting from the light-matter interaction operator [Eq. (12)] in the Coulomb gauge,

$$
\hat{V}_{\text {full }}(\mathbf{r}, \omega)=-e \frac{E_{\omega}}{2 \omega}(c \boldsymbol{\alpha} \cdot \boldsymbol{\epsilon}) e^{i(\mathbf{k} \cdot \mathbf{r}+\delta)},
$$

we find that the transformed operator reads

$$
\begin{aligned}
\hat{V}^{\prime}(\mathbf{r}, \omega) & =\hat{V}_{\text {full }}(\mathbf{r}, \omega)+i e \omega \chi(\mathbf{r}, \omega)+\frac{i e}{\hbar}[\chi(\mathbf{r}, \omega), \hat{\mathcal{H}}] \\
& =\frac{1}{2} i E_{\omega} \hat{T}_{\mathrm{mg}} e^{i \delta}=-\frac{1}{2} E_{\omega} \hat{T}_{\mathrm{mg}},
\end{aligned}
$$

where we have used Eq. (46) and recognize the effective interaction in the multipolar gauge [Eq. (38)]. The final form is obtained by setting the phase $\delta=\pi / 2$ in accordance with the phase convention
Eq. (24). This derivation thereby demonstrates that the change from velocity to length representation, Eq. (22) and its generalization in Eq. (50), is obtained by a gauge transformation.

\section{APPENDIX C: THE TRIVARIATE BETA FUNCTION}

In this appendix, we demonstrate the integral representation [Eq. (66)] of the trivariate beta function. We start from the integral representation of the gamma function, ${ }^{120}$

$$
\Gamma(a)=2 \int_{0}^{\infty} e^{-x^{2}} x^{2 a-1} d x,
$$

and consider the triple product

$\Gamma(a) \Gamma(b) \Gamma(c)=8 \int_{0}^{\infty} \int_{0}^{\infty} \int_{0}^{\infty} e^{-\left(x^{2}+y^{2}+z^{2}\right)} x^{2 a-1} y^{2 b-1} z^{2 c-1} d x d y d z$.

Noting that the integration is limited to the $(+,+,+)$ octant of Euclidean space, we switch to spherical coordinates

$$
\begin{aligned}
\Gamma(a) \Gamma(b) \Gamma(c)= & 8 \int_{0}^{\pi / 2} \int_{0}^{\pi / 2} \int_{0}^{\infty} e^{-r^{2}} r^{2(a+b+c)-3} \\
& \times e_{r ; x}^{2 a-1} e_{r ; y}^{2 b-1} e_{r: z}^{2 c-1} r^{2} \sin \theta d r d \theta d \phi \\
= & 4 \Gamma(a+b+c) \int_{0}^{\pi / 2} \int_{0}^{\pi / 2} e_{r ; x}^{2 a-1} e_{r ; y}^{2 b-1} e_{r ; z}^{2 c-1} \\
& \times \sin \theta d \theta d \phi,
\end{aligned}
$$

which leads directly to the introduction of the trivariate beta function and its integral representation as given in Eq. (66).

\section{APPENDIX D: SPECIFIC INTEGRALS OVER FULL AND TRUNCATED LIGHT-MATTER INTERACTION}

In the test case analyzed in Sec. IV B 2, the wave $\mathbf{k}$ and polarization $\boldsymbol{\epsilon}$ vectors are oriented along the $y$ - and $z$-axes, respectively, such that the full and truncated effective interaction operators at order $n$ are given by Eq. (72). We want to study the convergence of the underlying $\mathrm{AO}$-integrals over the truncated interaction toward the corresponding AO-integral over the full interaction. These involve only the scalar parts of the operators, so in practice, we study the expression

$$
\left\langle\chi_{\mu}\left|e^{+i k y}\right| \chi_{v}\right\rangle=\sum_{n=0}^{\infty} \frac{(i k)^{n}}{n !}\left\langle\chi_{\mu}\left|y^{n}\right| \chi_{v}\right\rangle
$$

where $\chi_{\mu}$ and $\chi_{\nu}$ are scalar basis functions. We shall limit attention to $p_{y}$ functions since the largest integrals in our study involved such basis functions with diffuse exponents.

The calculations presented in this paper are based on Cartesian Gaussian-type orbitals (CGTOs) [Eq. (75)]. With these basis functions, the volume integrals on both sides of Eq. (D1) factorize into integrals over the three Cartesian components. After elimination of common factors, Eq. (D1) reduces to

$$
\left\langle G_{j_{1}}^{\alpha_{1}}\left|e^{+i k y}\right| G_{j_{2}}^{\alpha_{2}}\right\rangle_{y}=\sum_{n=0}^{\infty} \frac{(i k)^{n}}{n !}\left\langle G_{j_{1}}^{\alpha_{1}}\left|y^{n}\right| G_{j_{2}}^{\alpha_{2}}\right\rangle_{y} ; \quad G_{j}^{\alpha}=N_{j}^{\alpha} y^{j} e^{-\alpha y^{2}} .
$$


The left-hand side integral corresponds to a Fourier transform. To evaluate the integral, we use the formula ${ }^{121}$

$$
\mathcal{F}\left[e^{-\alpha y^{2}}\right](k)=\int_{-\infty}^{+\infty} e^{-\alpha y^{2}} e^{i k y} d y=\sqrt{\frac{\pi}{\alpha}} e^{-k^{2} / 4 \alpha},
$$

as well as

$$
\left(-i \partial_{k}\right)^{n} \mathcal{F}[f(y)](k)=\int_{-\infty}^{+\infty} y^{n} f(y) e^{i k y} d y
$$

to obtain

$$
\left\langle G_{j_{1}}^{\alpha_{1}}\left|e^{+i k y}\right| G_{j_{2}}^{\alpha_{2}}\right\rangle_{y}=N_{j_{1}}^{\alpha_{1}} N_{j_{2}}^{\alpha_{2}} \sqrt{\frac{\pi}{\alpha_{1}+\alpha_{2}}}\left(\frac{i}{2 \sqrt{\alpha_{1}+\alpha_{2}}}\right)^{j_{1}+j_{2}} e^{-Q^{2}} H_{\left(j_{1}+j_{2}\right)}(Q)
$$

in terms of Hermite polynomials $H_{j}$ and the dimensionless parameter $Q$ [Eq. (78)]. For the right-hand side integral, we obtain

$$
\begin{aligned}
\left\langle G_{j_{1}}^{\alpha_{1}}\left|y^{n}\right| G_{j_{2}}^{\alpha_{2}}\right\rangle_{y}= & N_{j_{1}}^{\alpha_{1}} N_{j_{2}}^{\alpha_{2}} \frac{1}{2}\left[1+(-1)^{\left(j_{1}+j_{2}+n\right)}\right] \\
& \times\left(\alpha_{1}+\alpha_{2}\right)^{-\left(j_{1}+j_{2}+n+1\right) / 2} \Gamma\left(\frac{j_{1}+j_{2}+n+1}{2}\right),
\end{aligned}
$$

where we have used the integral representation of the gamma function

$$
\Gamma(a)=2 \int_{0}^{\infty} x^{2 a-1} e^{-x^{2}} d x .
$$

In our particular case, we have $j_{1}=j_{2}=1$, and so, one sees from the expression in square brackets of Eq. (D6) that only even $n=2 m$ contributions will be non-zero. Again eliminating common factors, we arrive at Eq. (77).

Further insight is provided by comparing with corresponding integrals obtained with Slater-type orbitals (STOs). We shall again limit attention to $p_{y}$ functions, which we express as

$$
S_{y}^{\zeta}=N_{\zeta} y \exp [-\zeta r] .
$$

In this case, factorization of integrals over Cartesian components is no longer possible. For the full interaction, we get

$$
\left\langle S_{y}^{\zeta_{1}}\left|e^{+i k y}\right| S_{y}^{\zeta_{2}}\right\rangle_{y}=32 \pi N_{\zeta_{1}} N_{\zeta_{2}} \bar{\zeta}\left(\bar{\zeta}^{2}-5 k^{2}\right)\left[\bar{\zeta}^{2}+k^{2}\right]^{-4} ; \quad \bar{\zeta}=\zeta_{1}+\zeta_{2},
$$

where we have used the Fourier transform ${ }^{121}$

$$
\mathcal{F}\left[e^{-\zeta r}\right](\boldsymbol{k})=8 \pi \frac{\zeta}{\left(\zeta^{2}+k^{2}\right)^{2}} .
$$

For the truncated interaction, we obtain

$$
\left\langle S_{y}^{\zeta_{1}}\left|y^{n}\right| S_{y}^{\zeta_{2}}\right\rangle=\frac{2 \pi}{n+3}\left[1-(-1)^{n+3}\right] N_{\zeta_{1}} N_{\zeta_{2}} \int_{0}^{\infty} r^{n+4} \exp [-\bar{\zeta} r] d r,
$$

where the expression in square brackets, coming from angular integration, again shows that only even $n=2 m$ contributions will be non-zero. The radial integral is found as

$$
\begin{aligned}
\int_{0}^{\infty} r^{2 m+4} \exp [-\bar{\zeta} r] d r & =\partial_{\bar{\zeta}}^{2 m+4} \int_{0}^{\infty} \exp [-\bar{\zeta} r] d r \\
& =(2 m+4) ! \bar{\zeta}^{-(2 m+5)} .
\end{aligned}
$$

After elimination of common factors, Eq. (D1) may, in this case, be expressed as

$$
\begin{aligned}
8\left(1-5 \tilde{Q}^{2}\right)\left[1+\tilde{Q}^{2}\right]^{-4} & =\sum_{m=0}^{\infty}(-1)^{m} \tilde{a}_{m} ; \\
\tilde{a}_{m} & =\tilde{Q}^{2 m}(2 m+4)(2 m+2)(2 m+1),
\end{aligned}
$$

where we have introduced the dimensionless variable

$$
\tilde{Q}=\frac{k}{\zeta_{1}+\zeta_{2}} \text {. }
$$

As in the case of CGTOs, the right-hand side has the form of an alternating series, but now, convergence becomes even more problematic since $\lim _{m \rightarrow \infty} \tilde{a}_{m}=\infty$. We also note the limit

$$
\lim _{m \rightarrow \infty} \frac{\tilde{a}_{m+1}}{\tilde{a}_{m}}=\tilde{Q}^{2} \lim _{m \rightarrow \infty} \frac{(m+3)(2 m+3)}{(m+1)(2 m+1)}=\tilde{Q}^{2},
$$

which is zero in the case of CGTOs. Numerically, we only find convergence of the right-hand side of Eq. (D13) for $\tilde{Q}<1$.

\section{REFERENCES}

${ }^{1}$ N. E. Christensen and B. O. Seraphin, Phys. Rev. B 4, 3321 (1971).

${ }^{2}$ P. Romaniello and P. L. de Boeij, J. Chem. Phys. 122, 164303 (2005).

${ }^{3}$ K. Glantschnig and C. Ambrosch-Draxl, New J. Phys. 12, 103048 (2010).

${ }^{4}$ F. Calvo, E. Pahl, M. Wormit, and P. Schwerdtfeger, Angew. Chem., Int. Ed. 52, 7583 (2013).

${ }^{\mathbf{5}}$ K. G. Steenbergen, E. Pahl, and P. Schwerdtfeger, J. Phys. Chem. Lett. 8, 1407 (2017).

${ }^{6}$ R. Ahuja, A. Blomqvist, P. Larsson, P. Pyykkö, and P. Zaleski-Ejgierd, Phys. Rev. Lett. 106, 018301 (2011).

${ }^{7}$ M. Göppert-Mayer, Ann. Phys. 401, 273 (1931).

${ }^{8}$ N. H. List, J. Kauczor, T. Saue, H. J. A. Jensen, and P. Norman, J. Chem. Phys. 142, 244111 (2015).

${ }^{9}$ P. V. Demekhin, J. Phys. B: At., Mol. Opt. Phys. 47, 025602 (2014).

${ }^{10}$ T. Katsouleas and W. B. Mori, Phys. Rev. Lett. 70, 1561 (1993).

${ }^{11}$ H. R. Reiss, Prog. Quantum Electron. 16, 1 (1992).

${ }^{12}$ H. Reiss, Phys. Rev. A 63, 013409 (2000).

${ }^{13}$ G. R. Shulman, Y. Yafet, P. Eisenberger, and W. E. Blumberg, Proc. Natl. Acad. Sci. U. S. A. 73, 1384 (1976).

${ }^{14}$ G. Dräger, R. Frahm, G. Materlik, and O. Brümmer, Phys. Status Solidi B 146, 287 (1988).

${ }^{15}$ T. Yamamoto, X-Ray Spectrom. 37, 572 (2008).

${ }^{16}$ S. D. George, T. Petrenko, and F. Neese, Inorg. Chim. Acta 361, 965 (2008).

${ }^{17}$ S. Bernadotte, A. J. Atkins, and C. R. Jacob, J. Chem. Phys. 137, 204106 (2012).

${ }^{18}$ L. D. Barron and C. G. Gray, J. Phys. A: Math., Nucl. Gen. 6, 59 (1973).

${ }^{19}$ L. D. Barron, Molecular Light Scattering and Optical Activity (Cambridge University Press, 2004).

${ }^{20}$ P. J. Lestrange, F. Egidi, and X. Li, J. Chem. Phys. 143, 234103 (2015).

${ }^{21}$ L. K. Sørensen, M. Guo, R. Lindh, and M. Lundberg, Mol. Phys. 115, 174 (2017).

${ }^{22}$ L. K. Sørensen, R. Lindh, and M. Lundberg, Chem. Phys. Lett. 683, 536 (2017).

${ }^{23}$ J. Lehtola, M. Hakala, A. Sakko, and K. Hämäläinen, J. Comput. Chem. 33, 1572 (2012).

${ }^{24}$ L. K. Sørensen, E. Kieri, S. Srivastav, M. Lundberg, and R. Lindh, Phys. Rev. A 99, 013419 (2019).

${ }^{25}$ N. H. List, T. Saue, and P. Norman, Mol. Phys. 115, 63 (2017).

${ }^{26}$ M. Khamesian, I. F. Galván, M. G. Delcey, L. K. Sørensen, and R. Lindh, Annual Reports in Computational Chemistry (Elsevier, 2019), Vol. 15, pp. 39-76.

${ }^{27}$ A. P. Markin and I. G. Kaplan, Dokl. Akad. Nauk SSSR 184, 66 (1969) (in Russian), available at http://mi.mathnet.ru/eng/dan/v184/i1/p66. 
${ }^{28}$ A. P. Markin and I. G. Kaplan, Sov. Phys. JETP 37, 216 (1973), available at http://www.jetp.ac.ru/cgi-bin/e/index/e/37/2/p216.

${ }^{29}$ G. M. Seabra, I. G. Kaplan, and J. V. Ortiz, J. Chem. Phys. 123, 114105 (2005).

${ }^{30}$ I. E. Brumboiu, O. Eriksson, and P. Norman, J. Chem. Phys. 150, 044306 (2019).

${ }^{31}$ M.-X. Wang, S.-G. Chen, H. Liang, and L.-Y. Peng, Chin. Phys. B 29, 013302 (2020).

${ }^{32}$ DIRAC, a relativistic ab initio electronic structure program, release DIRAC19, written by A. S. P. Gomes, T. Saue, L. Visscher, H. J. Aa. Jensen, and R. Bast, with contributions from I. A. Aucar, V. Bakken, K. G. Dyall, S. Dubillard, U. Ekström, E. Eliav, T. Enevoldsen, E. Faßhauer, T. Fleig, O. Fossgaard, L. Halbert, E. D. Hedegård, B. Heimlich-Paris, T. Helgaker, J. Henriksson, M. Iliaš, Ch. R. Jacob, S. Knecht, S. Komorovský, O. Kullie, J. K. Lærdahl, C. V. Larsen, Y. S. Lee, H. S. Nataraj, M. K. Nayak, P. Norman, G. Olejniczak, J. Olsen, J. M. H. Olsen, Y. C. Park, J. K. Pedersen, M. Pernpointner, R. di Remigio, K. Ruud, P. Sałek, B. Schimmelpfennig, B. Senjean, A. Shee, J. Sikkema, A. J. Thorvaldsen, J. Thyssen, J. van Stralen, M. L. Vidal, S. Villaume, O. Visser, T. Winther, and S. Yamamoto, available at https://doi.org/10.5281/zenodo.3572669, see also http://www.diracprogram.org, 2019.

${ }^{33}$ M. Gell-Mann, Il Nuovo Cimento 4, 848 (1956).

${ }^{34}$ K. Schwarzschild, Gött. Nach., Math.-Phys. Kl. 1903, 126, available at http://www.digizeitschriften.de/dms/img/?PID=GDZPPN002499665.

${ }^{35}$ T. Saue, Adv. Quantum Chem. 48, 383 (2005).

${ }^{36} \mathrm{P}$. Norman, K. Ruud, and T. Saue, Principles and Practices of Molecular Properties: Theory, Modeling and Simulations (Wiley, Hoboken, NJ, 2018).

${ }^{37} \mathrm{P}$. Atkins, Molecular Quantum Mechanics (Oxford University Press, 1996).

${ }^{38}$ C. Cohen-Tannoudji and D. Guéty-Odelin, Advances in Atomic Physics: An Overview (World Scientific, 2011), pp. 33-151.

${ }^{39}$ P. A. M. Dirac, The Principles of Quantum Mechanics, International Series of Monographs on Physics (Oxford, England) (Clarendon Press, Oxford, 1930), p. 232.

${ }^{40}$ G. Breit, Proc. Natl. Acad. Sci. U. S. A. 14, 553 (1928).

${ }^{41}$ T. Saue, ChemPhysChem 12, 3077 (2011).

${ }^{42}$ C. W. Bauschlicher, Jr. and S. R. Langhoff, Theor. Chim. Acta 79, 93 (1991).

${ }^{43}$ T. Saue and H. J. A. Jensen, J. Chem. Phys. 111, 6211 (1999).

${ }^{44}$ D. H. Kobe, Am. J. Phys. 50, 128 (1982).

${ }^{45}$ A. M. Stewart, J. Phys. A: Math. Gen. 32, 6091 (1999).

${ }^{46} \mathrm{~T}$. Saue, in Relativistic Electronic Structure Theory. Part 1. Fundamentals, edited by P. Schwerdtfeger (Elsevier, Amsterdam, 2002). p. 332.

${ }^{47} \mathrm{~F}$. Bloch, in W. Heisenberg und die Physik unserer Zeit, edited by F. Bopp (Vieweg \& Sohn, Braunschweig, 1961).

${ }^{48}$ P. Lazzeretti, Theor. Chim. Acta 87, 59 (1993).

${ }^{49}$ W. E. Brittin, W. R. Smythe, and W. Wyss, Am. J. Phys. 50, 693 (1982).

${ }^{50}$ B. -S. K. Skagerstam, Am. J. Phys. 51, 1148 (1983).

${ }^{51}$ C. Cohen-Tannoudji, J. Dupont-Roc, and G. Grynberg, Photons et Atomes (Savoirs Actuels, New York, 1987).

${ }^{52}$ J. D. Jackson and L. B. Okun, Rev. Mod. Phys. 73, 663 (2001).

${ }^{53}$ R. Bast, J. Jusélius, and T. Saue, Chem. Phys. 356, 187 (2009).

${ }^{54}$ V. I. Lebedev, USSR Comput. Math. Math. Phys. 15, 44 (1975).

${ }^{55}$ V. I. Lebedev, USSR Comput. Math. Math. Phys. 16, 10 (1976).

${ }^{56}$ V. I. Lebedev, Sib. Math. J. 18, 99 (1977).

${ }^{57}$ V. I. Lebedev and A. L. Skorokhodov, Russ. Acad. Sci. Dokl. Math. 45, 587 (1992), available at https://zbmath.org/?q=an\%3A0795.41026.

${ }^{58}$ V. I. Lebedev, Russ. Acad. Sci. Dokl. Math. 50, 283 (1995), available at https://zbmath.org/?q=an\%3A0863.41018.

${ }^{59}$ V. I. Lebedev and D. N. Laikov, Dokl. Math. 59, 477-481 (1999), available at https://zbmath.org/? $\mathrm{q}=\mathrm{an} \% 3 \mathrm{~A} 0960.65029$, angular quadrature parameters available from http://server.ccl.net/cca/software/SOURCES/FORTRAN/LebedevLaikov-Grids/index.shtml.

${ }^{60}$ E. A. Kearsley and J. T. Fong, J. Res. Nat. Bur. Stand. B. Math. Sci. 79B, 49 (1975). ${ }^{61}$ D. L. Andrews and T. Thirunamachandran, J. Chem. Phys. 67, 5026 (1977).

${ }^{62}$ D. L. Andrews and W. A. Ghoul, J. Phys. A: Math. Gen. 14, 1281 (1981).

${ }^{63}$ D. L. Andrews and N. P. Blake, J. Phys. A: Math. Gen. 22, 49 (1989).

${ }^{64}$ D. H. Friese, M. T. P. Beerepoot, and K. Ruud, J. Chem. Phys. 141, 204103 (2014).
${ }^{65}$ J.-H. Ee, D.-W. Jung, U.-R. Kim, and J. Lee, Eur. J. Phys. 38, 025801 (2017).

${ }^{66} \mathrm{H}$. Weyl, The Classical Groups (Princeton University Press, 1939).

${ }^{67}$ P. G. Hodge, Am. Math. Monthly 68, 793 (1961).

${ }^{68}$ H. Jeffreys, Math. Proc. Cambridge Philos. Soc. 73, 173 (1973), Jeffreys acknowledges previous work by M. Pastori.

${ }^{69}$ G. F. Smith, Tensor 19, 79 (1968).

${ }^{70}$ R. S. Rivlin, J. Ration. Mech. Anal. 4, 681 (1955).

${ }^{71}$ See http://oeis.org/A005043 for information about Motzkin sums.

${ }^{72}$ M. Stener, G. Fronzoni, and M. de Simone, Chem. Phys. Lett. 373, 115 (2003).

${ }^{73}$ C. South, A. Shee, D. Mukherjee, A. K. Wilson, and T. Saue, Phys. Chem. Chem. Phys. 18, 21010 (2016).

${ }^{74}$ J. P. Perdew, K. Burke, and M. Ernzerhof, Phys. Rev. Lett. 77, 3865 (1996).

${ }^{75}$ C. Adamo and V. Barone, J. Chem. Phys. 110, 6158 (1999).

${ }^{76}$ K. G. Dyall, J. Phys. Chem. A 113, 12638 (2009).

${ }^{77}$ K. G. Dyall, Theor. Chem. Acc. 131, 1217 (2012).

${ }^{78}$ L. Visscher, Theor. Chem. Acc. 98, 68 (1997).

${ }^{79}$ L. Visscher and K. G. Dyall, At. Data Nucl. Data Tables 67, 207 (1997).

${ }^{80}$ A. D. Becke, Phys. Rev. A 38, 3098 (1988).

${ }^{81}$ J. P. Perdew, Phys. Rev. B 33, 8822 (1986); Erratum, 34, 7406 (1986).

${ }^{82}$ E. Van Lenthe and E. J. Baerends, J. Comput. Chem. 24, 1142 (2003).

${ }^{83}$ J.-M. Lévy-Leblond, Commun. Math. Phys. 6, 286 (1967).

${ }^{84}$ W. J. Hehre, R. Ditchfield, and J. A. Pople, J. Chem. Phys. 56, 2257 (1972).

${ }^{85}$ P. C. Hariharan and J. A. Pople, Theor. Chim. Acta 28, 213 (1973).

${ }^{86}$ M. M. Francl, W. J. Pietro, W. J. Hehre, J. S. Binkley, M. S. Gordon, D. J. DeFrees, and J. A. Pople, J. Chem. Phys. 77, 3654 (1982).

${ }^{87}$ V. A. Rassolov, J. A. Pople, M. A. Ratner, and T. L. Windus, J. Chem. Phys. 109, 1223 (1998).

${ }^{88}$ M. J. Frisch, G. W. Trucks, H. B. Schlegel, G. E. Scuseria, M. A. Robb, J. R. Cheeseman, G. Scalmani, V. Barone, G. A. Petersson, H. Nakatsuji, X. Li, M. Caricato, A. V. Marenich, J. Bloino, B. G. Janesko, R. Gomperts, B. Mennucci, H. P. Hratchian, J. V. Ortiz, A. F. Izmaylov, J. L. Sonnenberg, D. WilliamsYoung, F. Ding, F. Lipparini, F. Egidi, J. Goings, B. Peng, A. Petrone, T. Henderson, D. Ranasinghe, V. G. Zakrzewski, J. Gao, N. Rega, G. Zheng, W. Liang, M. Hada, M. Ehara, K. Toyota, R. Fukuda, J. Hasegawa, M. Ishida, T. Nakajima, Y. Honda, O. Kitao, H. Nakai, T. Vreven, K. Throssell, J. A. Montgomery, Jr., J. E. Peralta, F. Ogliaro, M. J. Bearpark, J. J. Heyd, E. N. Brothers, K. N. Kudin, V. N. Staroverov, T. A. Keith, R. Kobayashi, J. Normand, K. Raghavachari, A. P. Rendell, J. C. Burant, S. S. Iyengar, J. Tomasi, M. Cossi, J. M. Millam, M. Klene, C. Adamo, R. Cammi, J. W. Ochterski, R. L. Martin, K. Morokuma, O. Farkas, J. B. Foresman, and D. J. Fox, gaussian 16, Revision C.01, Gaussian, Inc.Wallingford, CT, 2016.

${ }^{89}$ T. Glaser, B. Hedman, K. O. Hodgson, and E. I. Solomon, Acc. Chem. Res. 33, 859 (2000).

${ }^{90}$ E. I. Solomon, B. Hedman, K. O. Hodgson, A. Dey, and R. K. Szilagyi, Coord. Chem. Rev. 249, 97 (2005).

${ }^{91}$ S. DeBeer George, P. Brant, and E. I. Solomon, J. Am. Chem. Soc. 127, 667 (2005).

${ }^{92}$ J. A. Bearden and A. F. Burr, Rev. Mod. Phys. 39, 125 (1967).

${ }^{93}$ Mathematica, Version 11.3, Wolfram Research, Inc., Champaign, IL, 2018.

${ }^{94}$ T. Helgaker and P. R. Taylor, HERMIT, A Molecular Integral Code, University of Oslo, Oslo, Norway, 1986.

${ }^{95}$ R. Bast, H. J. A. Jensen, and T. Saue, Int. J. Quantum Chem. 109, 2091 (2009).

${ }^{96}$ H. Sambe, Phys. Rev. A 7, 2203 (1973).

${ }^{97}$ O. Christiansen, P. Jørgensen, and C. Hättig, Int. J. Quantum Chem. 68, 1 (1998).

${ }^{98}$ N. Rösch, Chem. Phys. 80, 1 (1983).

${ }^{99} \mathrm{~T}$. Saue and H. J. A. Jensen, J. Chem. Phys. 118, 522 (2003).

${ }^{100} \mathrm{P}$. Jørgensen and J. Linderberg, Int. J. Quantum Chem. 4, 587 (1970).

${ }^{101}$ P. Jorgensen, Annu. Rev. Phys. Chem. 26, 359 (1975).

${ }^{102}$ M. Casida, in Recent Advances in Density Functional Methods: Part I, edited by

D. P. Chong (World Scientific, Singapore, 1995), p. 155.

${ }^{103}$ D. Peng, W. Zou, and W. Liu, J. Chem. Phys. 123, 144101 (2005). 
${ }^{104} \mathrm{~F}$. Wang, T. Ziegler, E. van Lenthe, S. van Gisbergen, and E. J. Baerends, J. Chem. Phys. 122, 204103 (2005).

${ }^{105}$ D. H. Kobe, Am. J. Phys. 51, 105 (1983).

${ }^{106}$ D. H. Kobe and R. Dale Gray, Il Nuovo Cimento B 86, 155 (1985).

${ }^{107}$ E. A. Power and T. Thirunamachandran, Phys. Lett. A 87, 449 (1982).

${ }^{108}$ Molecules in Laser Fields, edited by A. D. Bandrauk (Marcel Dekker, 1994).

${ }^{109}$ V. Chernyak and S. Mukamel, Chem. Phys. 198, 133 (1995).

110 A. Salam, Phys. Rev. A 56, 2579 (1997).

${ }^{11}$ R. Anzaki, Y. Shinohara, T. Sato, and K. L. Ishikawa, Phys. Rev. A 98, 063410 (2018).

${ }^{112}$ V. Fock, Z. Phys. 39, 226 (1926).

${ }^{113}$ V. A. Fock - Selected Works: Quantum Mechanics and Quantum Field Theory, edited by L. Faddeev, L. Khalfin, and I. Komarov (CRC Press, 2019).
${ }^{114}$ E. A. Power and S. Zienau, Philos. Trans. R. Soc., A 251, 427 (1959).

${ }^{115}$ P. W. Atkins, R. G. Woolley, and C. A. Coulson, Philos. Trans. R. Soc., A $\mathbf{3 1 9}$, 549 (1970).

${ }^{116}$ R. G. Woolley and C. A. Coulson, Philos. Trans. R. Soc., A 321, 557 (1971).

${ }^{117}$ E. Rousseau and D. Felbacq, Sci. Rep. 7, 11115 (2017).

${ }^{118}$ D. L. Andrews, G. A. Jones, A. Salam, and R. G. Woolley, J. Chem. Phys. 148, 040901 (2018).

${ }^{119}$ M. Babiker, R. Loudon, and G. W. Series, Philos. Trans. R. Soc., A 385, 439 (1983).

${ }^{120}$ G. B. Arfken, H. J. Weber, and F. E. Harris, Mathematical Methods for Physicists (Academic Press, Amsterdam, 2013).

${ }^{121}$ R. S. Strichartz, A Guide to Distribution Theory and Fourier Transforms (CRC Press, Boca Raton, 1993), pp. 41, 50. 